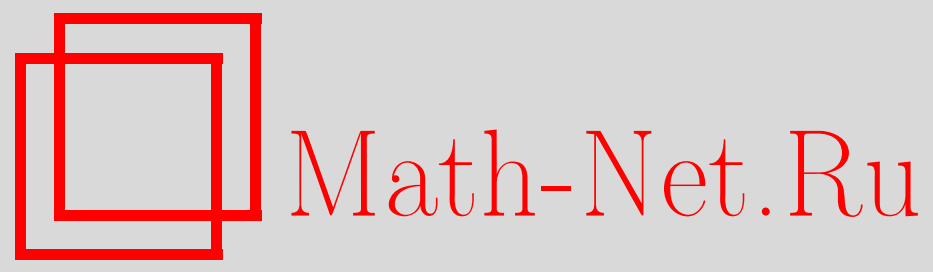

Е. В. Горбатов, Стандартный базис полиномиального идеала над коммутативным артиновым цепным кольцом, Дискрет. матем., 2004, том 16, выпуск 1, 52-78

DOI: https://doi.org/10.4213/dm142

Использование Общероссийского математического портала Math-Net.Ru подразумевает, что вы прочитали и согласны с пользовательским соглашением http://www . mathnet.ru/rus/agreement

Параметры загрузки:

IP: 54.224 .187 .69

26 апреля 2023 г., 10:18:11

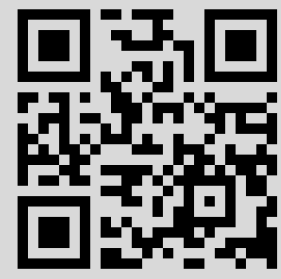




\title{
Стандартный базис полиномиального идеала над коммутативным артиновым цепным кольцом
}

\author{
() 2004 г. $\quad$ Е. В. Горбатов
}

\begin{abstract}
Строится стандартный базис идеала кольца полиномов $R[X]=R\left[x_{1}, \ldots, x_{k}\right]$ над коммутативным артиновым цепным кольцом $R$, обобщающий понятие базиса Гребнера полиномиального идеала над полем. При этом используется предложенное в работах Д. А. Михайлова и А. А. Нечаева понятие старшего члена полинома, учитывающее специфику кольца $R$; в отличие от этих работ, предлагаемые конструкции основываются на понятии схемы симплификации, предложенной В. Н. Латышевым. Доказано, что всякая каноническая система образующих (КСО), построенная в работах Д. А. Михайлова и А. А. Нечаева, является стандартным базисом специального вида. Введено понятие $S$-полинома и на его основе построен алгоритм, находящий стандартный базис идеала и КСО идеала. Определяются минимальный и редуцированный стандартные базисы, приводятся характеризующие их условия. Доказано, что при естественном эпиморфизме $v: R[X] \rightarrow \bar{R}[X]$, где $\bar{R}=R / \operatorname{rad}(R)$, базис Гребнера $\chi$ полиномиального идеала над полем вычетов $\bar{R}$ поднимается до стандартного базиса той же мощности в $R[X]$ тогда и только тогда, когда идеал $(\chi)$ является образом некоторого идеала $I \triangleleft R[X]$, являющегося свободным $R$-модулем.

Работа поддержана Российским фондом фундаментальных исследований, грант 02-01-00218, и грантом НШ-1910.2003.1 Президента РФ для поддержки ведущих научных школ.
\end{abstract}

\section{1. Введение}

Любое построение теории стандартных базисов идеалов кольца $R[X]=R\left[x_{1}, \ldots, x_{k}\right]$ полиномов (над каким-либо кольцом $R$ ) сводится к определению некоторого алгоритма редуцирования полинома $F \in R[X]$ с помощью системы полиномов $\chi \subseteq R[X]$. При этом само определение редукции основывается на понятии старшего члена полинома, которое, в свою очередь, подразумевает введение некоторого упорядочения на одночленах. Это упорядочение (часто неявно) продолжается до порядка на всем кольце $R[X]$. Наличие этого порядка обеспечивает сходимость процесса редуцирования за конечное число шагов (при этом необходимо, чтобы редукции уменьшали полиномы и сам порядок удовлетворял условию обрыва убывающих цепей).

Формальной основой при моделировании описанной выше ситуации является развитая в [4] техника схем симплификация. В данной работе мы последовательно используем эту технику (необходимые сведения приводятся в разделе 2). 
Если основное кольцо $R$ есть поле, то задача определения старшего члена, редукций, стандартного базиса (базиса Гребнера-Ширшова) решается однозначно при фиксированном допустимом порядке (см., например, [5, 6]). В изучаемом случае, когда $R$ - коммутативное артиново цепное кольцо, существует несколько подходов к решению этой задачи. В [7] старший член полинома определялся, как и в классическом случае кольца полиномов над полем, исходя из допустимого порядка на мономах (без учета коэффициентов из $R$ ). Основанный на этом определении алгоритм редуцирования привел к построению стандартного базиса, названного минимальным сильным базисом Гребнера. В [8] для случая, когда $\boldsymbol{R}$ - кольцо Галуа, использовалось то же определение старшего члена полинома, но другой алгоритм редуцирования. Там также был построен стандартный базис полиномиального идеала. Базисы из [7] и [8] позволяют решать проблему вхождения полинома $F$ в идеал $I \triangleleft R[X]$. Заметим, что для случая одной переменной минимальный сильный базис Гребнера из [7] был построен гораздо раньше в [1] и был назван канонической системой образующих (КСО) полиномиального идеала. Вместе с тем работа [7] не содержит ссылок на эти результаты.

В отличие от вышеуказанных работ, в [1], а затем и в $[2,3]$, использовалось другое упорядочение членов полинома, учитывающее специфику кольца $R$, и в связи с этим другое понятие старшего члена. Например, старшим членом полинома $2 x+1 \in \mathbf{Z}_{4}[x]$ согласно [1] будет 1 , а не $2 x$, как в $[5,7,8]$. Как следует из предложения 3 , такое упорядочение одночленов является естественным. Результатом работ [1-3] явилось построение КСО для произвольного полиномиального идеала. В данной работе при построении стандартного базиса используется такое же упорядочение членов полинома. Таким образом, КСО и предлагаемый здесь стандартный базис являются согласованными с нормой кольца $R$ (см. (6)); любая КСО есть стандартный базис в нашем смысле, удовлетворяющий некоторым дополнительным условиям (см. раздел 5).

Необходимо отметить, что в работах [9-11] также использовалось упорядочение членов полинома, учитывающее специфику кольца $R$. Предлагаемое в указанных работах понятие градуированной структуры позволяет взглянуть с общей позищии на различные подходы к построению стандартных базисов. Тем не менее, описываямая в нашей работе конструкция не вкладывается в эту классификацию (см. замечание 4); рассмотренный в данной работе более узкий класс колец коэффициентов позволяет расширить возможности построения стандартных базисов и глубже изучить их структуру (см., например, теорему 1).

Как показано в [1-3], КСО в ряде случаев более эффективна при решении прикладных задач, она позволяет не только решать проблему вхождения, но и вычислять мощность факторкольца $R[X] / I$, строить систему образующих семейства линейных рекуррент, вычислять период идеала (в случае одной переменной) и решать некоторые другие задачи (см. теорему 4.11 в [2]).

Предлагаемый в данной работе формализованный подход, основанный на технике схем симплификации, привел к определению стандартного базиса более общему чем КСО из [3] (сравнение стандартного базиса и КСО приводится в разделе 4). Для стандартного базиса установлены некоторые свойства, аналогичные свойствам КСО (см. предложение 17). В [1-3] не было предложено эффективной процедуры для построения КСО. В данной работе водится понятие $S$-полинома, и на его основе строится алгоритм, вычисляющий стандартный базис и КСО полиномиального идеала по произвольной системе порождающих (см. Алгоритм 1). Также были получены новые характеризации цилиндрических идеалов (см. теорему 5), что позволило решить часть поставленных в [3] задач.

Стандартный базис обладает свойствами по форме весьма похожими на свойства ба- 
зисов Гребнера полиномиальных идеалов над полем. Например, минимальный и редуцированный стандартные базисы (см. предложение 17 и определение 18) характеризуются условиями, подобными классическим (см. теорему 3 ).

Получено продвижение в решении выдвинутой в [2] проблемы о поднятии базиса Гребнера, а именно, доказано, что базис Гребнера $\chi$ полиномиального идеала над полем вычетов $\bar{R}=R / \operatorname{rad}(R)$ поднимается до стандартного базиса той же мощности в $R[X]$ при естественном эпиморфизме $\nu: R[X] \rightarrow \bar{R}[X]$ тогда и только тогда, когда идеал $(\chi)$ является образом некоторого идеала $I \triangleleft R[X]$, являющегося свободным $R$-модулем (см. теорему 6).

\section{2. Схемы симплификации}

Ввиду неоднозначности терминология, дадим определения используемых понятий и объектов.

Множество натуральных чисел, как обычно, обозначаем $\mathbf{N}=\{1,2,3, \ldots\}$, множество натуральных чисел с нулем $-\mathbf{N}_{0}=\{0,1,2, \ldots\}$, отрезок целых чисел $-\{m, \ldots, n\}=$ $\{i \in \mathbf{Z} \mid m \leqslant i \leqslant n\}$.

Запись $A \subseteq B$ означает, что $A$ является подмножеством $B$. Если $A$ - собственное подмножество $B$ (то есть $A$ есть подмножество $B$ и $A \neq B$ ), то пишем $A \subset B$.

Мы говорим, что бинарное отношение $\preccurlyeq$ на $M$ задает порядок на $M$ или что $(M, \preccurlyeq)$ является упорядоченным множеством, если это отношение рефлексивно, транзитивно и антисимметрично (в этом случае также употребляют термин частично упорядоченное множество). Если любые два элемента из $M$ сравнимы относительно порядка $\preccurlyeq$, мы говорим, что $(M, \preccurlyeq)$ - линейно упорядоченное множество, а порядок $\preccurlyeq$ называем линейным. Наконец, линейный порядок $\preccurlyeq$, удовлетворяющий условию минимальности (условию обрыва убывающих цепочек), называем полным и при таком условии говорим, что $(M, \preccurlyeq)$ - вполне упорядоченное множество.

Наше построение стандартного базиса полиномиального идеала будет существенно использовать язык схем симплификации из [4]. Далее приводятся необходимые сведения из этой теории.

Определение 1. Пусть $(M, \preccurlyeq)$ - непустое упорядоченное множество с условием минимальности и $S \subseteq M^{M}$. Тройка $\subseteq=(M, \preccurlyeq, S)$ называется схемой симплификации на $M$, если выполнено условие

$$
\forall m \in M \forall s \in S: \quad s(m) \preccurlyeq m .
$$

При этом порядок $\preccurlyeq$ называется порядком сложности. Элементы из $S$ называются (одношаговыми) симплификаторами или (элементарными) редукциями.

Определение 2. Пусть $\subseteq=(M, \preccurlyeq, S)-$ схема симплификации. Говорят, что $m \in M-$ нормальный или редуцированный элемент (относительно $\subseteq$ ), если $s(m)=m$ для всех $s \in S$, иначе элемент $m$ называется редуцируемым. Совокупность всех нормальных элементов обозначается $N_{\subseteq}$.

Отметим, что $N_{\varsigma} \neq \varnothing$, поскольку $\preccurlyeq$ удовлетворяет условию минимальности.

Обозначим через $\hat{S}$ подполугруппу в полугруппе $M^{M}$ (с композищией функций в качестве умножения), порожденную $S \cup\left\{1_{M}\right\}$, то есть

$$
\hat{S}=\left|S, 1_{M}\right\rangle=\left\{s_{1} \circ \ldots \circ s_{n} \mid(n \in \mathbf{N}) \&\left(\forall i \in\{1, \ldots, n\} s_{i} \in S\right)\right\} \cup\left\{1_{M}\right\} .
$$


Элементы из $\hat{S}$ называются редукциями или симплификаторами (не одношаговыми).

Определение 3. Пусть $\mathfrak{S}=(M, \preccurlyeq, S)-$ схема симплификации. Для любого элемента $m \in M$ множество нормальных форм определяется равенством

$$
\operatorname{Nor}_{S}(m)=\hat{S}(m) \cap N_{\Im} \text {. }
$$

Любой элемент из $\operatorname{Nor}_{5}(m)$ называется нормальной формой $m$. Так как порядок $\preccurlyeq$ удовлетворяет условию минимальности, множество нормальных форм непусто. Для произвольного подмножества $L \subseteq M$ также определяется

$$
\operatorname{Nor}_{\Im}(L)=\bigcup_{l \in L} \operatorname{Nor}_{\Im}(l)
$$

Например, $\operatorname{Nor}_{5}(M)=N_{\Im}-$ множество всех нормальных элементов.

Определение 4. Пусть $\subseteq=(M, \preccurlyeq, S)-$ схема симплификации. Тогда если $\left|\operatorname{Nor}_{\varsigma}(m)\right|=1$, то говорят, что $m \in M$ обладает канонической формой. В этом случае элемент $\operatorname{Can}_{\subseteq}(m) \in M$, определяемый из равенства $\operatorname{Nor}_{5}(m)=\left\{\operatorname{Can}_{5}(m)\right\}$, называется канонической формой $m$.

Множество всех элементов из $M$, обладающих канонической формой, обозначается $C_{\Xi}$.

Если $C_{\subseteq}=M$, то говорят, что $\subseteq-$ схема симплификации с канонизацией.

Очевидно, что $N_{\Im} \subseteq C_{\subseteq}$. Поэтому мы можем рассматривать Саnฐ как отображение из $C_{5}$ в $C_{\Xi}$.

Для краткости, когда из контекста ясно, какая схема симплификации рассматривается, мы будем писать $N$, Nor, Can и $C$ вместо $N_{\Im}, N_{5}, \operatorname{Can}_{5}$ и $C_{5}$, соответственно.

Лемма 1. Пусть $\mathfrak{S}=(M, \preccurlyeq, S)-$ схема симплификачии. Тогда для любых элементов $m_{1}, \ldots, m_{t}$ из $M$ существует симплификатор $\sigma \in \hat{S}$ такой, что $\sigma\left(m_{i}\right) \in \operatorname{Nor}\left(m_{i}\right)$ для всех $i \in\{1, \ldots, t\}$.

Доказательство. Используем индукщию по $t$. При $t=1$ лемма утверждает, что любой элемент из $M$ можно привести к нормальной форме. Проведем шаг индукщии $t \rightarrow t+1$, $t \geqslant 1$. Существует симплификатор $\sigma_{1} \in \hat{S}$ такой, что элементы $\sigma_{1}\left(m_{1}\right), \ldots, \sigma_{1}\left(m_{t}\right)$ нормальны. Также существует редукщия $\sigma_{2} \in \hat{S}$, приводящая $\sigma_{1}\left(m_{t+1}\right)$ к нормальной форме. Тогда $\sigma=\sigma_{2} \circ \sigma_{1}$ приводит все элементы $m_{1}, \ldots, m_{t+1}$ к нормальной форме.

Определение 5. Пусть $R$ - некоторое кольцо и $M_{R}$ - правый $R$-модуль. Схема симплификации $\subseteq=\left(M_{R}, \preccurlyeq, S\right)$ называется $R$-линейной, если $S \subseteq \operatorname{End}\left(M_{R}\right)$.

Предложение 1. Пусть $\subseteq=\left(M_{R}, \preccurlyeq, S\right)-R$-линейная схема симплификачии. Тогда $C=C_{\Im}$ и $N=N_{\Im}-$ подмодули в $M_{R}$. Отображение Can: $C \rightarrow C$ является проектором, mo ecmb Can $\in \operatorname{End}\left(C_{R}\right) u \operatorname{Can}^{2}=\operatorname{Can} u$, cmaлo быmb,

$$
C=\operatorname{Im}(\text { Can }) \oplus \operatorname{Ker}(\mathrm{Can})=N \oplus \operatorname{Ker}(\mathrm{Can}) .
$$

Доказательство. Пусть $c_{1}, c_{2} \in C, \quad r_{1}, r_{2} \in R$ и $c=c_{1} r_{1}+c_{2} r_{2}$. Допустим, что $\sigma(c) \in \operatorname{Nor}(c)$ для некоторого симплификатора $\sigma \in \hat{S}$. Тогда $\sigma(c)=\sigma\left(c_{1}\right) r_{1}+\sigma\left(c_{2}\right) r_{2}$. 
По лемме 1 существует симплификатор $\rho \in \hat{S}$ такой, что и $\rho\left(\sigma\left(c_{1}\right)\right)$, и $\rho\left(\sigma\left(c_{2}\right)\right)$ нормальны. Для любого такого симплификатора

$$
\sigma(c)=\rho \sigma(c)=\rho \sigma\left(c_{1}\right) r_{1}+\rho \sigma\left(c_{2}\right) r_{2}=\operatorname{Can}\left(c_{1}\right) r_{1}+\operatorname{Can}\left(c_{2}\right) r_{2} \text {. }
$$

Значит, $c$ обладает единственной нормальной формой, то есть $c \in C$. Таким образом, $C-$ подмодуль в $M_{R}$.

Более того, мы доказали, что $\operatorname{Can}(c)=\operatorname{Can}\left(c_{1}\right) r_{1}+\operatorname{Can}\left(c_{2}\right) r_{2}$, то есть $\operatorname{Can} \in \operatorname{End}\left(C_{R}\right)$.

Наконец, $\operatorname{Can}(n)=n$ для любого элемента $n \in N$, поэтому $N=\operatorname{I} \ln (\operatorname{Can})$, и следовательно, $N$ - подмодуль в $M_{R}$.

\section{3. Схема симплификации на полиномах}

Всюду далее $R$ - коммутативное артиново локальное кольцо главных идеалов. Структура идеалов кольца $R$ имеет вид

$$
R>\pi R>\pi^{2} R>\ldots>\pi^{n-1} R>\pi^{n} R=0,
$$

где $n-$ индекс нильпотентности радикала Джекобсона $J=\operatorname{rad}(R), n>1$ и $\pi$ - произвольный элемент из $J \backslash J^{2}$. Элемент $\pi$ предполагается фиксированным. Зафиксируем также множество переменных $X=\left\{x_{1}, \ldots, x_{k}\right\}, k \geqslant 1$.

Определим, как и в [1], нормы элемента $r \in R$, пллинома $F \in R[X]$ и подмножества $\chi \subset R[X]$ равенствами

$$
\begin{aligned}
\|r\| & =\max \left\{i \in\left\{0,1, \ldots, n \mid r \in \pi^{i} R\right\},\right. \\
\|F\| & =\max \left\{i \in\left\{0,1, \ldots, n \mid F \in \pi^{i} R[X]\right\},\right. \\
\|\chi\| & =\max \left\{i \in\left\{0,1, \ldots, n \mid \chi \subseteq \pi^{i} R[X]\right\} .\right.
\end{aligned}
$$

Главной задачей этого раздела является построение схемы симплификации $\mathbb{S}_{\chi}=\left(R[X], \preccurlyeq, S_{\chi}\right)$.

Основным множеством схемы симплификации $\mathscr{S}_{\chi}$ является алгебра полиномов $R[X]=R\left[x_{1}, \ldots, x_{k}\right]$ от $k$ коммутируюших переменных над кольцом $R$.

Определение порядка сложности $\preccurlyeq$ требует более детального рассмотрения.

Определение 6. Пусть $(U, \cdot)$ - полугруппа и $\preccurlyeq-$ порядок на $U$. Тройка $(U, \cdot, \preccurlyeq)$ называется упорядоченной полугруппой, если

$$
\forall a, b, c \in U: \quad(a \preccurlyeq b) \Longrightarrow(a c \preccurlyeq b c) \&(c a \preccurlyeq c b) .
$$

Если $\preccurlyeq-$ линейный (полный) порядок на $U$, то говорят, что $(U, \cdot, \preccurlyeq)-$ линейно (вполне) упорядоченная полугруппа.

Пусть $[X]=\left[x_{1}, \ldots, x_{k}\right]$ - полугруппа коммутативных мономов над $X$. Очевидно, что $([X], \cdot, \mid)$ - упорядоченная полугруппа (здесь | обозначает отношение делимости), изоморфная упорядоченной полугрупше $\left(\mathbf{N}_{0}^{k},+, \leqslant\right)$, где + означает покомпонентное сложение, а порядок $\leqslant$ получается из обычного порядка на $\mathbf{N}_{0}$ по формуле

$$
\left(a_{1}, \ldots, a_{k}\right) \leqslant\left(b_{1}, \ldots, b_{k}\right) \Longleftrightarrow a_{1} \leqslant b_{1}, \ldots, a_{k} \leqslant b_{k} .
$$




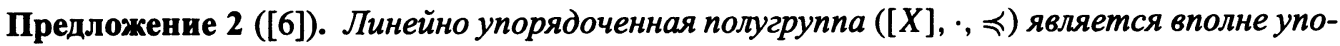
рядоченной полугруппой, если и только если $1 \preccurlyeq$ и для всех $и \in[X]$. В этом случае порядок $\preccurlyeq$ называется допустимым.

При заданном допустимом порядке $\preccurlyeq$ на $[X]$ любой ненулевой полином $F \in R[X]$ может быть представлен в виде

$$
F=a_{1} u_{1}+\ldots+a_{m} u_{m}
$$

где $a_{1}, \ldots, a_{m}$ - ненулевые элементы из $R$ и $u_{1}, \ldots, u_{m}$ - мономы, такие, что $u_{1} \succ \ldots \succ u_{m}$. Исходя из этого представления, можно определить старший моном $u_{1}$, старший член $a_{1} u_{1}$ и старший коэффициент $a_{1}$ полинома $F$. Именно эти понятия использовались в большинстве предыдущих работ при построении редукщий на полиномах и стандартных базисов идеалов (см. $[12,5,7,8])$. В отличие от этого подхода, в [1], а затем и в $[2,3]$ было по существу использовано другое упорядочение членов полинома, учитывающее специфику кольца $R$, что привело к построению стандартного базиса (названного авторами канонической системой образующих (КСО)), более эффективного при решении ряда прикладных задач (см. [1-3]).

Здесь мы опишем формализованную конструкцию, позволяющую взглянуть с общих позищий на построения работ [1-3] и получить дальнейшие продвижения в решении поставленных там задач. Для этого вместо полугруппы мономов [X] рассмотрим следующий объект, учитывающий специфику кольца $R$.

Определение 7. Полугруппой $\pi$-мономов называется подполугруппа

$$
[\pi, X]=\left[\pi, x_{1}, \ldots, x_{k}\right]=\left\{\pi^{a} u \mid a \in\{0,1, \ldots, n-1 ; u \in[X]\} \cup\{0\}\right.
$$

полугруппы $(R[X], \cdot)$. Формально

$$
[\pi, X]=\left\langle x_{0}, x_{1}, \ldots, x_{k} \mid x_{i} x_{j}=x_{j} x_{i}, x_{0}^{n} x_{l}=x_{0}^{n}, i, j, l \in\{0,1, \ldots, k\}\right\rangle .
$$

Очевидно, что полугруппа $[X]$ является подполугруппой полугруппы $[\pi, X]$. Пусть на $[X]$ задан некоторый допустимый порядок $\preccurlyeq$. Тогда на $[\pi, X]$ можно ввести два порядка $\preccurlyeq-\mathbf{и ~} \preccurlyeq+$.

Определим $\preccurlyeq-$, полагая

$$
\pi^{a} u \preccurlyeq-\pi^{b} v \Longleftrightarrow\{a>b \text { или } a=b \text { и } u \preccurlyeq v\},
$$

где $a, b \in\{0,1, \ldots, n-1\}, u, v \in[X]$, и $0 \preccurlyeq-U$ для всех $U \in[\pi, X]$.

Симметрично определяется порядок $\preccurlyeq+$ :

$$
\pi^{a} u \preccurlyeq+\pi^{b} v \Longleftrightarrow\{a<b \text {, или } a=b \text { и } u \preccurlyeq v\},
$$

где $a, b \in\{0,1, \ldots, n-1\}, u, v \in[X]$, и $U \preccurlyeq+0$ для всех $U \in[\pi, X]$.

Легко видеть, что каждый из порядков $\preccurlyeq-и \preccurlyeq+$ превращает $[\pi, X]$ во вполне упорядоченную полугруппу, в которой $([X], \cdot, \preccurlyeq)$ - упорядоченная подполугруппа. Более того, как показывает следующее утверждение, других порядков с таким свойством нет.

Предложение 3. Пусть на $[\pi, X]$ задан порядок $\leqslant$ такой, что $([\pi, X], \cdot, \leqslant)-$ вполне упорядоченная полугруппа $u \preccurlyeq-$ ограничение $\leqslant$ на $[X]$. Тогда $\leqslant$ совпадает $c \preccurlyeq-$ или $\preccurlyeq+$. 
Доказательство. Возможны два случая $\pi<1$ и $1<\pi$.

Допустим, что $\pi<1$. Тогда

$$
0<\pi^{n-1}<\ldots<\pi<1,
$$

и значит, для любого монома $u \in[X]$ и любого $a \in\{0,1, \ldots, n-1\}$

$$
0<\pi^{a} u \text {. }
$$

Далее, если $a, b \in\{0,1, \ldots, n-1\}$ и $a>b$, то

$$
\pi^{a} u<\pi^{b} v
$$

для любых мономов $u, v \in[X]$. В противном случае $\pi^{b} v \leqslant \pi^{a} u$ и $\pi^{n-a+b} v \leqslant 0$, что противоречит (11). Из условий (11) и (12) следует, что исходный порядок $\leqslant$ совпадает с $\preccurlyeq-$.

Во втором случае, когда $1<\pi$, аналогичные рассуждения показывают, что порядок $\leqslant$ совпадает $\mathrm{c} \preccurlyeq+$.

Для наших целей подходит лишь порядок $\preccurlyeq-$, при котором 0 является самым младшим $\pi$-мономом (в то время как порядок $\preccurlyeq+$ таков, что $U \preccurlyeq+0$ для любого $U \in[\pi, X]$ ).

Ввиду сказанного выше, естественным продолжением порядка $\preccurlyeq$ на $[\pi, X]$ будем называть порядок $\preccurlyeq-$, и поскольку в дальнейшем порядок $\preccurlyeq+$ использоваться не будет, для обозначения рассматриваемого продолжения будем употреблять прежний символ $\preccurlyeq$. Далее полагаем, что вместе с порядком $\preccurlyeq$ заданы и порядки $\succcurlyeq, \prec, \succ$, при этом, например,

$$
a \succ b \Longleftrightarrow(a \preccurlyeq b) \&(a \neq b) \text {. }
$$

Легко проверяется следующее утверждение.

Предложение 4. Пусть $\preccurlyeq-$ допустимый порядок на $[X]$. Тогда его естественное продолжение на $[\pi, X]$ обладает следующими свойствами:

$$
\begin{aligned}
\|U\|>\|V\| & \Longrightarrow U \prec V, \\
U \preccurlyeq V, \quad V W=0 & \Longrightarrow U W=0, \\
U \prec V, \quad V W \neq 0 & \Longrightarrow U W \prec V W,
\end{aligned}
$$

для любых л-мономов $U, V, W \in[\pi, X]$.

Пусть $M$ - некоторое непустое множество и $M_{\text {fin }}-$ множество всех конечных подмножеств $M$ :

$$
M_{\text {fin }}=\left\{X \subseteq M|| X \mid<\aleph_{0}\right\} .
$$

Предположим, что на $M$ задан некоторый линейный порядок $\preccurlyeq$. Он индуцирует линейный порядок $\preccurlyeq$ на $M_{\text {fin }}$. А именно, пусть $A=\left\{a_{1}, \ldots, a_{m}\right\}$ и $B=\left\{b_{1}, \ldots, b_{n}\right\}-$ непустые множества из $M_{\text {fin }}$, причем $a_{1} \succ \ldots \succ a_{m}$ и $b_{1} \succ \ldots \succ b_{n}$, положим

$$
A \preccurlyeq B \Longleftrightarrow\left\{\begin{array}{l}
m \leqslant n \text { и } a_{i}=b_{i}, i \in\{1, \ldots, m\}, \text { или } \\
\exists k \leqslant \min \{m, n\}: a_{1}=b_{1}, \ldots, a_{k-1}=b_{k-1}, a_{k} \prec b_{k} .
\end{array}\right.
$$

Для любого $C \in M_{\text {fin }}$ по определению полагаем, что $\varnothing \preccurlyeq C$. Иными словами, построенный на $M_{\text {fin }}$ порядок $\preccurlyeq$ сводится к лексикографическому сравнению слов, получающихся из множеств выписыванием их элементов по убыванию.

Нам не удалось найти опубликованного доказательства следующего, по-видимому, хорошо известного утверждения, и мы приводим его здесь для полноты изложения. 
Предложение 5. Упорядоченное множество ( $\left.M_{\mathrm{fin}}, \preccurlyeq\right)$ является вполне упорядоченным тогда и только тогда, когда $(M, \preccurlyeq)$ - вполне упорядоченное множество.

Доказательство. Пусть $\left(M_{\text {fin }}, \preccurlyeq\right)$ - вполне упорядоченное множество. Рассмотрим вложение

$$
\mu: M \ni a \mapsto\{a\} \in M_{\text {fin }}
$$

Ясно, что

$$
a \preccurlyeq b \Longleftrightarrow \mu(a) \preccurlyeq \mu(b),
$$

так что $(M, \preccurlyeq)$ является вполне упорядоченным множеством.

Обратно, пусть $(M, \preccurlyeq)$ - вполне упорядоченное множество. Допустим, что существует строго убывающая последовательность $\left\{S_{i}\right\}_{i \in \mathrm{N}}$ множеств из $M_{\text {fin }}$. Ясно, что последовательность $\left\{S_{i}\right\}_{i \in \mathrm{N}}$ не содержит пустого множества. Пусть $\max (S)$ - наибольший элемент непустого множества $S \in M_{\text {fin }}$ относительно порядка $\preccurlyeq$ на $M$. Тогда $\left\{\max \left(S_{i}\right)\right\}_{i \in \mathbf{N}}$ - убывающая (не обязательно строго убывающая) последовательность элементов из $M$; если эта последовательность постоянна, перейдем к рассмотрению строго убывающей последовательности множеств $\left\{S_{i} \backslash\left\{\max \left(S_{i}\right)\right\}\right\}_{i \in N}$; применяя эту процедуру нужное число раз, получим строго убывающую последовательность множеств $\left\{S_{i}^{\prime}\right\}_{i \in \mathrm{N}}$, такую, что $\left\{\max \left(S_{i}^{\prime}\right)\right\}_{i \in \mathrm{N}}$ - непостоянная последовательность. Поэтому в $\left\{S_{i}^{\prime}\right\}_{i \in \mathbf{N}}$ можно выбрать подпоследовательность $\left\{S_{i}^{\prime \prime}\right\}_{i \in \mathbf{N}}$ такую, что $\max \left(S_{1}^{\prime \prime}\right) \succ \max \left(S_{2}^{\prime \prime}\right)$. Итак, по любой строго убывающей последовательности множеств $\left\{S_{i}\right\}_{i \in \mathbf{N}}$ можно построить строго убывающую последовательность $\left\{S_{i}^{(1)}\right\}_{i \in \mathrm{N}}$ такую, что $\max \left(S_{1}\right) \succcurlyeq \max \left(S_{1}^{(1)}\right) \succ \max \left(S_{2}^{(1)}\right)$. Применяя указанную процедуру к последовательности $\left\{S_{i}^{(1)}\right\}_{i \geqslant 2}$, получим строго убывающую последовательность $\left\{S_{i}^{(2)}\right\}_{i \geqslant 2}$ такую, что $\max \left(S_{2}^{(1)}\right) \succcurlyeq \max \left(S_{2}^{(2)}\right) \succ \max \left(S_{3}^{(2)}\right)$. По индукции построим семейство последовательностей $\left\{S_{i}^{(k)}\right\}_{i \geqslant k}, k \in \mathbf{N}$, таких, что

$$
\max \left(S_{k+1}^{(k)}\right) \succcurlyeq \max \left(S_{k+1}^{(k+1)}\right) \succ \max \left(S_{k+2}^{(k+1)}\right), \quad k \in \mathbf{N},
$$

$\left\{\max \left(S_{k}^{(k)}\right)\right\}_{k \in \mathbf{N}}$ - строго убывающая последовательность элементов из $M$. Полученное противоречие завершает доказательство.

Пусть $F \in R[X]$ и $u \in[X]$. Элемент из $R$, являющийся коэффициентом при мономе $u$ в полиноме $F$, обозначим через $\operatorname{Cf}(F, u)$.

Носителем многочлена $F \in R[x]$ назовем множество

$$
\operatorname{supp}(F)=\{u \in[X] \mid \operatorname{Cf}(F, u) \neq 0\} .
$$

Носитель $\operatorname{supp}(F)$ является элементом $[X]_{\text {fin }}$.

Назовем $\pi$-носителем многочлена $F \in R[x]$ множество

$$
\operatorname{Supp}(F)=\left\{U \in[\pi, X] \mid U=\pi^{\|\operatorname{Cf}(F, u)\|} u, u \in \operatorname{supp}(F)\right\} .
$$

Множество $\operatorname{Supp}(F)$ является элементом $[\pi, X]_{\text {fin }}$, причем $0 \notin \operatorname{Supp}(F)$.

Пусть $\preccurlyeq-$ некоторый допустимый порядок на $[X]$. Его естественное продолжение на $[\pi, X]$ будет полным порядком. Далее, согласно предложению 5 , порядок $\preccurlyeq$ на $[\pi, X]_{\text {fin }}$, индуцированный порядком $\preccurlyeq$ на $[\pi, X]$, также будет полным. Наконец, для любых $F, G \in R[X]$ по определению полагаем

$$
F \prec G \Longleftrightarrow \operatorname{Supp}(F) \prec \operatorname{Supp}(G),
$$


при этом

$$
F \preccurlyeq G \Longleftrightarrow(F \prec G) \vee(F=G)
$$

Замечание 1. Отметим, что построенный порядок $\preccurlyeq$ в общем случае не будет линейным. Например, в кольце $Z_{4}[x]$ полиномы $x$ и $3 x$ несравнимы (при единственном допустимом порядке $\preccurlyeq$ на $[x])$.

Тем не менее, упорядоченное множество $(R[X], \preccurlyeq)$ удовлетворяет условию минимальности, так как порядок $\preccurlyeq$ на $[\pi, X]_{\text {fin }}$ полный.

Итак, мы продолжили допустимый порядок $\preccurlyeq \mathrm{c}[X]$ на $R[X]$ по цепочке

$$
[X],[\pi, X],[\pi, X]_{\text {fin }}, R[X] \text { : }
$$

В дальнейшем, когда задан некоторый допустимый порядок на $[X]$, порядки на $[\pi, X]$, $[\pi, X]_{\text {fin }}$ и $R[X]$ также предполагаются заданными.

Приступим к построению последнего элемента нашей схемы симплификации - семейства редукщий $S_{\chi}$.

Пусть на $[X]$ задан некоторый допустимый порядок $\preccurlyeq$. Пусть также дан некоторый полином $F \in R[X] \backslash 0$. Тогда $\pi$-носитель $\operatorname{Supp}(F)$ непуст, и в нем относительно порядка $\preccurlyeq$ существует наибольший $\pi$-моном $V_{0}=\pi^{a_{0}} v_{0} \in[\pi, X]$. Введем обозначения

$$
\begin{aligned}
\operatorname{Lm}(F) & =v_{0} & & - \text { ведущий моном } F, \\
\operatorname{LC}(F) & =\operatorname{Cf}\left(F, v_{0}\right) & & \text { - ведущий коэффициент } F, \\
\operatorname{Lt}(F) & =\operatorname{LC}(F) \operatorname{Lm}(F) & & \text { - ведущий член } F, \\
\operatorname{LM}(F) & =\pi^{\|\operatorname{LC}(F)\|} \operatorname{Lm}(F)=V_{0} & & \text { - ведущий } \pi \text {-моном } F .
\end{aligned}
$$

Для произвольного $\chi \subseteq R[X]$ будем полагать

$$
\operatorname{Lm}(\chi)=\{\operatorname{Lm}(G) \mid G \in \chi\}
$$

Аналогичные соглашения принимаем и для остальных введенных выше функций.

Замечанне 2. Для любого полинома $F$ верны равенства

$$
\|F\|=\|\mathrm{LC}(F)\|=\|\operatorname{Lt}(F)\|=\|\mathrm{LM}(F)\| .
$$

С помощью предложения 4 легко доказывается следующее утверждение.

Предложкене 6. Пусть даны два полинома $F, G \in R[X]$ такие, что $F G \neq 0$, тогда выполняются соотношения

$$
\begin{aligned}
\operatorname{Lm}(F G) & =\operatorname{Lm}(F) \operatorname{Lm}(G), \\
\operatorname{LC}(F G) & =\operatorname{LC}(F) \operatorname{LC}(G), \\
\operatorname{Lt}(F G) & =\operatorname{Lt}(F) \operatorname{Lt}(G) \\
\operatorname{LM}(F G) & =\operatorname{LM}(F) \operatorname{LM}(G)
\end{aligned}
$$

Положим по определению $\mathrm{LC}(0)=\operatorname{Lt}(0)=\mathrm{LM}(0)=0$ (значение $\operatorname{Lm}(0)$ считаем неопределенным). При этом все формулы, кроме первой, из предложения 6 остаются верными для любых $F, G \in R[X]$. 
Пусть даны полином $G \in R[X] \backslash 0$ с ведущим коэффищиентом $a=\operatorname{LC}(G)$ и моном $u \in[X]$. Определим редукцию $r_{G, u}: R[X] \rightarrow R[X]$, ассоциированную с парой $(G, u)$, следующим образом: для любого $F \in R[X]$, если $c=\operatorname{Cf}(F, u \operatorname{Lm}(G))$, то

$$
r_{G, u}(F)= \begin{cases}F, & \text { если }\|c\|<\|a\|, \\ F-b u G, & \text { если }\|c\| \geqslant\|a\|, c=b a .\end{cases}
$$

Отметим, что данное определение не зависит от выбора элемента $b$. Действительно, для другого элемента $b^{\prime} \in R$ такого, что $c=b^{\prime} a$ справедливы соотношения

$$
\left(b-b^{\prime}\right) \operatorname{LC}(G)=\left(b-b^{\prime}\right) a=0 \Longrightarrow\left(b-b^{\prime}\right) G=0 \Longrightarrow F-b u G=F-b^{\prime} u G .
$$

Для любого монома $u \in[X]$ полагаем $r_{0, u}=1_{R[X]}$.

Заметим что $r_{G, u}(F)=F$, в точности если $\|c\|<\|a\|$ или $c=0$ (в последнем случае $b u G=0)$.

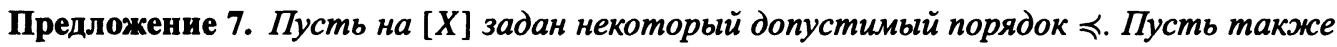
даны полином $G \in R[X]$ и моном $u \in[X]$. Тогда $r_{G, u}(F) \preccurlyeq F$ для любого многочлена $F \in R[X]$, в частности, $\|F\| \preccurlyeq\left\|r_{G, u}(F)\right\|$.

Доказательство. В обозначениях (21), если $\|c\|<\|a\|$ или $c=0$, то $r_{G, u}(F)=F$ и утверждение верно. Остается рассмотреть ситуацию, когда $\|c\| \geqslant\|a\|$ и $c \neq 0$ (и следовательно, $G \neq 0$ ).

Полином $F$, сгруппировав надлежащим образом его члены, можно записать в виде

$$
F=F_{1}+c u \operatorname{Lm}(G)+F_{0},
$$

где элементы $\operatorname{Supp}\left(F_{1}\right)$ старше $c u \operatorname{Lm}(G)$, а элементы $\operatorname{Supp}\left(F_{0}\right)$ младше $c u \operatorname{Lm}(G)$.

Далее, разобьем члены полинома $b u G$ на четыре группы:

$$
b u G=c u \operatorname{Lm}(G)+G_{2}+G_{1}+G_{0},
$$

так, чтобы выполнялись соотношения

$$
\begin{gathered}
\operatorname{supp}\left(G_{0}\right) \subseteq \operatorname{supp}\left(F_{0}\right), \quad \operatorname{supp}\left(G_{1}\right) \subseteq \operatorname{supp}\left(F_{1}\right), \\
\operatorname{supp}\left(G_{2}\right) \cap\left(\operatorname{supp}\left(F_{0}\right) \cup \operatorname{supp}\left(F_{1}\right)\right)=\varnothing
\end{gathered}
$$

Справедливы равенства

$$
r_{G, u}(F)=F-b u G=\left(F_{1}-G_{1}\right)+\left(F_{0}-G_{0}-G_{2}\right) .
$$

Покажем что

$$
\operatorname{Supp}\left(F_{1}-G_{1}\right)=\operatorname{Supp}\left(F_{1}\right)
$$

Действительно, пусть $a v$ и $b v-$ одночлены из $F_{1}$ и $G_{1}$ соответственно. Так как $a v \succ c u \operatorname{Lm}(G) \succ b v$, то $\|a\| \leqslant\|c\| \leqslant\|b\|$. В последней цепочке может встретиться не более одного равенства (в противном случае были бы справедливы соотношения $v>u \operatorname{Lm}(G) \succ v)$. Значит, $\|a\|<\|b\|$, откуда и вытекает совпадение указанных $\pi$-носителей. 
Так как $\|a+b\| \geqslant \min (\|a\|,\|b\|)$, все элементы $\operatorname{Supp}\left(F_{0}-G_{0}-G_{2}\right)$ младше $c u \operatorname{Lm}(G)$. Из сказанного выше и из равенств

$$
\begin{aligned}
\operatorname{Supp}(F) & =\operatorname{Supp}\left(F_{1}\right) \cup\left\{\pi^{\|c\|} u \operatorname{Lm}(G)\right\} \cup \operatorname{Supp}\left(F_{0}\right), \\
\operatorname{Supp}\left(r_{G, u}(F)\right) & =\operatorname{Supp}\left(F_{1}\right) \cup \operatorname{Supp}\left(F_{0}-G_{0}-G_{2}\right)
\end{aligned}
$$

следует, что $\operatorname{Supp}\left(r_{G, u}(F)\right) \prec \operatorname{Supp}(F)$, и значит, $r_{G, u}(F) \prec F$.

Пусть $\chi \subseteq R[X]$ и

$$
S_{\chi}=\left\{r_{G, u} \mid G \in \chi, u \in[X]\right\}
$$

- множество редукций, отвечающих системе многочленов $\chi$. Тогда, в силу утверждения 7 , $\mathbb{S}_{\chi}=\left(R[X], \preccurlyeq, S_{\chi}\right)$ есть схема симплификации. Более того, очевидно, что справедливо следующее утверждение.

Предложение 8. Для любых полинома $G \in R[X] \backslash 0$ и монома $u \in[X]$

$$
r_{G, u} \in \operatorname{End}_{R}\left(\pi^{\|G\|} R[X]\right) .
$$

Из утверждения 8 вытекает, что тройка $\left(\pi^{d} R[X], \preccurlyeq, S_{\chi}\right)$, где $d \geqslant \max \{\|G\| \mid G \in \chi\}$, является $R$-линейной схемой симплификации (см. определение 5).

Замечание 3. Если $\|G\|=0$, то $\mathrm{LC}(G) \in R^{*}$, и мы можем задать редукцию формулой

$$
r_{G, u}(F)=F-\operatorname{Cf}(F, u \operatorname{Lm}(G)) \operatorname{LC}(G)^{-1} u G .
$$

Отскда непосредственно видно, что $r_{G, u} \in \operatorname{End}_{R}(R[X])$. Однако, даже если $\|G\| \neq 0$, то для любого элемента $a \in R^{*}$ имеет место соотношение

$$
r_{G, u}(a F)=a r_{G, u}(F) .
$$

Замечание 4. Роль полугрупшы (моноида) $[\pi, X]$ в наших рассмотрениях аналогична роли основного моноида $\Gamma$, используемого при построении градуированных структур в [9-11]. Вместе с тем полугруппа $[\pi, X]$ не является полугруппой с сокращением, тогда как моноид $\Gamma$, согласно определению градуированной структуры, должен обладать этим свойством. Таким образом, предлагаемая здесь конструкщия не покрывается теорией градуированных структур.

\section{4. $\quad S$-полиномы и стандартные базисы}

Здесь, по аналогии с известными результатами для полиномов над полями (см., например, $[5,6])$, вводится понятие $S$-полинома и на его основе исследуется метод построения стандартного базиса полиномиального идеала по его произвольной системе образующих.

Зафиксируем некоторый допустимый порядок $\preccurlyeq$ на полугруппе мономов $[X]$.

Определение 8. Будем говорить, что полином $F \in R[X]$ обладает представлением относительно системы полиномов $\chi \subseteq R[X]$, если

$$
F=\sum_{i=1}^{m} a_{i} u_{i} G_{i},
$$


где $a_{i} \in R, u_{i} \in[X]$ и $G_{i} \in \chi$. Параметром представления (26) назовем $\pi$-моном

$$
W=\max _{\preccurlyeq}\left\{\operatorname{LM}\left(a_{i} u_{i} G_{i}\right) \mid i \in\{1, \ldots, m\}\right\} .
$$

Если $m=0$, параметр представления неопределен.

Очевидно следующее утверждение.

Предложение 9. Если полином $F \in R[X] \backslash 0$ обладает представлением (26) с параметром $W$, mo

$$
\mathrm{LM}(F) \preccurlyeq W .
$$

Определение 9. Представление (26) называется $H$-представлением (относительно системы полиномов $\chi$ ), если $F=0$ или $\mathrm{LM}(F)=W$, где $W$ - параметр этого представления.

Определение 10. Полином $F \in R[X] \backslash 0$ назовем $\pi$-унитарным, если

$$
\mathrm{LC}(F)=\pi^{\|F\|} \text {. }
$$

Для любого полинома $F \in R[X]$ существуют элемент $a \in R^{*}$ и такой $\pi$-унитарный полином $\stackrel{\circ}{F}$, что $F=a \stackrel{\circ}{F}$. Полином $\stackrel{\circ}{F}$ определяется по $F$ однозначно. Действительно, пусть $F=a^{\prime} F^{\prime}$, где $a^{\prime} \in R^{*}$ и $F^{\prime}-\pi$-унитарный полином. Тогда $\stackrel{\circ}{F}=b F^{\prime}, b \in R^{*}$, и значит, $\pi^{\|F\|}=\mathrm{LC}(\stackrel{\circ}{F})=\mathrm{LC}(b) \operatorname{LC}\left(F^{\prime}\right)=b \pi^{\|F\|}$. Поэтому

$$
\stackrel{\circ}{F}=b F^{\prime}=b \pi^{\|F\|} G^{\prime}=\pi^{\|F\|} G^{\prime}=F^{\prime} .
$$

В представлении $F=a \stackrel{\circ}{F}$ элемент $a \in R^{*}$ определен однозначно по модулю $\pi^{n-\|F\|}$.

Введем естественным образом отношение делимости на полугруппе $[\pi, X]$. Ясно, что $([\pi, X], \mid)$ - упорядоченное множество, причем для любых двух $\pi$-мономов $U, V \in[\pi, X]$ существует их точная верхняя грань $\operatorname{lcm} U V$ и точная нижняя грань $\operatorname{gcd} U V$ (таким образом $([\pi, X], \mid)$ - решетка). Отметим также, что

$$
U \mid V \Longrightarrow\|U\| \leqslant\|V\| \text {. }
$$

Пусть $F, G \in R[X] \backslash 0$ и $\operatorname{LM}(F)=\pi^{a} u, \operatorname{LM}(G)=\pi^{b} v$, где $a, b \in\{0,1, \ldots, n-1\}$ и $u, v \in[X]$. Пусть $w=\operatorname{gcd}(u, v)-$ наибольший общий делитель $u, v \in[X]$ и $c=\max \{a, b\}$. Существуют мономы $u^{\prime}, v^{\prime} \in[X]$ такие, что $u=w u^{\prime}$ и $v=w v^{\prime}$. S-полиномом от $F$ и $G$ называется полином

$$
S(F, G)=\pi^{c-a} v^{\prime} \stackrel{\circ}{F}-\pi^{c-b} u^{\prime} \stackrel{\circ}{ } .
$$

Равенство (29) можно рассматривать как представление полинома $S(F, G)$ относительно системы $\chi=\{\stackrel{\circ}{F}, \stackrel{\leftrightarrow}{G}\}$. Параметром этого представления будет $\pi$-моном $\pi^{c} u^{\prime} v^{\prime} w$, он называется начальным параметром $S$-полинома $S(F, G)$.

Для удобства полагаем $S(F, 0)=S(0, G)=0$ для любых полиномов $F, G \in R[X]$, причем параметр в этом случае считается равным 0 .

Отметим, что для любых полиномов $F, G \in R[X]$ начальный параметр $S$-полинома равен наименьшему общему кратному $\operatorname{lcm} \operatorname{LM}(F) \operatorname{LM}(G)$.

Определение 11. Назовем непустую систему полиномов $\chi \subseteq R[X] \pi$-однородной, если $\|G\|=\|\chi\|$ для любого $G \in \chi$. 
Любую систему $\chi \subseteq R[X]$ можно представить в виде объединения $\pi$-однородных подсистем:

$$
\chi=\chi_{0} \cup \chi_{1} \cup \ldots \cup \chi_{t}
$$

Положим $\alpha_{s}=\left\|\chi_{s}\right\|, s \in\{0,1, \ldots, t\}$. Используя разложение (30), всегда будем считать, что

$$
0 \leqslant \alpha_{0}<\alpha_{1}<\ldots<\alpha_{t}
$$

В $R[X]$ найдутся такие системы полиномов $\psi_{s}, s \in\{0,1, \ldots, t\}$, что

$$
\pi^{\alpha_{s}} \psi_{s}=\chi_{s}, \quad s \in\{0,1, \ldots, t\}
$$

Также, для удобства записи, положим $\chi_{t+1}=\psi_{t+1}=\varnothing$ и $\alpha_{t+1}=\left\|\chi_{t+1}\right\|=n$.

Следующая теорема является аналогом леммы о композиции (см., например, $[5,6])$ из теории базисов Гребнера над полями.

Tеорема 1. Пусть $\chi$ - непустая система полиномов из идеала $I$ кольча $R[X]$, представленная в виде объединения $\pi$-однородных подсистем (30) со свойством (31), пусть также выбраны системы полиномов $\psi_{s}, s \in\{0,1, \ldots, t+1\}$, такие, что выполняется условие (32). Тогда следуючие утверждения эквивалентны:

(a) $\operatorname{LM}(I)=\operatorname{LM}(\chi)[\pi, X]-$ идеал полугруппы $[\pi, X]$, порожденный $\mathrm{LM}(\chi)$,

(b) $\forall F \in I: \exists G \in \chi \quad \mathrm{LM}(G) \mid \mathrm{LM}(F)$,

(c) $\forall F \in I: \operatorname{Nor}_{\mathscr{S}_{x}}(F)=0$,

(d) $\forall F \in I: \operatorname{Nor}_{\mathscr{S}_{\chi}}(F) \ni 0$,

(е) любой полином $F \in I$ обладает $H$-представлением относительно $\chi$,

(f) $I=(\chi) u \forall G_{1}, G_{2} \in \chi: \operatorname{Nor}_{\mathscr{S}_{\chi}}\left(S\left(G_{1}, G_{2}\right)\right)=0$,

(g) $I=(\chi) u \forall G_{1}, G_{2} \in \chi: \operatorname{Nor}_{\mathscr{S}_{\chi}}\left(S\left(G_{1}, G_{2}\right)\right) \ni 0$,

(h) $I=(\chi)$ u $\forall G_{1}, G_{2} \in \chi$ либо $S\left(G_{1}, G_{2}\right)=0$, либо $S$-полином $S\left(G_{1}, G_{2}\right)$ обладает представлением с параметром, меньшим его начаіьного параметра,

(i) $I=(\chi) u$

$$
\begin{aligned}
\forall j \in\{0,1, \ldots, t\} \forall F & \in \pi^{\alpha_{j}} R[X]: N_{1}, N_{2} \in \operatorname{Nor}_{\mathscr{\Theta}_{x_{0}} \cup \ldots \cup x_{j}}(F) \\
& \Longrightarrow N_{1}-N_{2} \in\left(\pi^{\alpha_{j+1}} \psi_{0} \cup \ldots \cup \pi^{\alpha_{j+1}} \psi_{j} \cup \chi_{j+1} \cup \ldots \cup \chi_{t}\right) .
\end{aligned}
$$

Доказательство. Ясно, что (a)

Докажем, что (b) $\Rightarrow(\mathrm{c})$. Пусть $H \in \operatorname{Nor}_{\mathscr{\leftrightarrow}_{x}}(F)$, тогда $H=0$. В противном случае, ввиду пункта (b), полином $H \in I$ был бы редуцируем.

Импликация (c) $\Rightarrow$ (d) очевидна. 
Докажем, что (d) $\Rightarrow$ (e). Очевидно, что если $F=0$, то утверждение верно. Пусть $F \neq 0$. Согласно (d), существует последовательность редукщий $r_{1}, \ldots, r_{m} \in_{\chi}, m \geqslant 1$, такая, что

$$
\begin{aligned}
F=F_{0} & \stackrel{r_{1}}{\longmapsto} F_{1} \stackrel{r_{2}}{\longmapsto} \ldots \stackrel{r_{m}}{\longmapsto} F_{m}=0, \\
& F_{0} \succ F_{1} \succ \ldots \succ F_{m} .
\end{aligned}
$$

Пусть $r_{i}=r_{G_{i}, u_{i}}$, где $G_{i} \in \chi, u_{i} \in[X]$ при $i \in\{1, \ldots, m\}$. Справедливо равенство

$$
F-a_{1} u_{1} G_{1}-\ldots-a_{m} u_{m} G_{m}=F_{m}=0
$$

что дает нам представление $F$ относительно $\chi$. Остается доказать, что параметр этого представления равен $\mathrm{LM}(F)$.

Так как $r_{i}\left(F_{i-1}\right) \neq F_{i-1}$ для любого $i \in\{1, \ldots, m\}$, то $\operatorname{LM}\left(a_{i} u_{i} G_{i}\right) \preccurlyeq \operatorname{LM}\left(F_{i-1}\right)$ для любого $i \in\{1, \ldots, m\}$. В силу (33)

$$
\mathrm{LM}\left(F_{0}\right) \succcurlyeq \mathrm{LM}\left(F_{1}\right) \succcurlyeq \ldots \succcurlyeq \operatorname{LM}\left(F_{m-1}\right) .
$$

Значит, $\mathrm{LM}\left(a_{i} u_{i} G_{i}\right) \preccurlyeq \mathrm{LM}(F)$ для любого $i \in\{1, \ldots, m\}$. Поэтому параметр представления $W$ не превосходит $\operatorname{LM}(F)$ (относительно порядка $\preccurlyeq$ ). Учитывая предложение 9 , получаем, что $W=\mathrm{LM}(F)$.

Докажем, что (e) $\Rightarrow(\mathrm{b})$. Пусть $F \in I$. Если $F=0$, то $\mathrm{LM}(F)=0$ делится на $\mathrm{LM}(G)$ для любого $G \in \chi$. Считаем, что $F \neq 0$. Согласно (e), $F$ обладает $H$-представлением вида (26). По определению $H$-представления

$$
\begin{aligned}
\operatorname{LM}(F) & =\max \left\{\operatorname{LM}\left(a_{i} u_{i} G_{i}\right) \mid i \in\{1, \ldots, m\}\right\} \\
& =\operatorname{LM}\left(a_{i_{0}} u_{i_{0}} G_{i_{0}}\right)=\operatorname{LM}(F)=\operatorname{LM}\left(a_{i_{0}} u_{i_{0}}\right) \operatorname{LM}\left(G_{i_{0}}\right)
\end{aligned}
$$

и значит, $\operatorname{LM}\left(G_{i_{0}}\right) \mid \operatorname{LM}(F)$.

Импликация (c) $\Rightarrow$ (f) очевидна.

Импликация (f) $\Rightarrow(\mathrm{g})$ очевидна.

Доказательство того, что $(\mathrm{g}) \Rightarrow(\mathrm{h})$ аналогично доказательству справедливости импликации $(\mathrm{d}) \Rightarrow(\mathrm{e})$.

Докажем, что (h) $\Rightarrow$ (e). Допустим, что условие (e) не выполнено. Тогда существует полином $F \in I \backslash 0$, не имеющий $H$-представления. Тем не менее, ввиду того, что $I=(\chi)$, полином $F$ имеет представление относительно системы $\chi$. Из всех возможных представлений (26) полинома $F$ выберем имеющие наименьший параметр $W \in[\pi, X], \mathbf{a}$ из отобранных возьмем представление такое, что мощность множества

$$
T=\left\{i \in\{1, \ldots, m\} \mid \operatorname{LM}\left(a_{i} u_{i} G_{i}\right)=W\right\}
$$

минимальна. Так как $F$ не обладает $H$-представлением, то $\operatorname{LM}(F) \prec W$, и значит, $|T| \geqslant 2$. Меняя, если необходимо, нумерацию, можно считать, что $1,2 \in T$. Справедливы равенства $W=\operatorname{LM}\left(a_{1} u_{1} G_{1}\right)=\operatorname{LM}\left(a_{2} u_{2} G_{2}\right)$, поэтому $\operatorname{LM}\left(G_{1}\right) \mid W$ и $\operatorname{LM}\left(G_{2}\right) \mid W$. Значит, $W=U W_{0}$, где $W_{0}=\operatorname{lcm} \operatorname{LM}\left(G_{1}\right) \operatorname{LM}\left(G_{2}\right)$ и $U \in[\pi, X]$. Далее, существуют $\pi$-мономы $V_{1}, V_{2} \in[\pi, X]$ такие, что $W_{0}=V_{1} \operatorname{LM}\left(G_{1}\right), W_{0}=V_{2} \operatorname{LM}\left(G_{2}\right)$. Согласно (h), $S$-полином $S\left(G_{1}, G_{2}\right)=V_{1} \dot{G}_{1}-V_{2} \dot{G}_{2}$ обладает представлением

$$
S\left(G_{1}, G_{2}\right)=\sum_{i=1}^{m} a_{i}^{\prime} u_{i}^{\prime} G_{i}
$$


с параметром, меньшим исходного параметра $W_{0}$ (Добавляя, в случае необходимости, нулевые слагаемые, можно считать, что множества индексов в представлениях (26) и (35) совпадают), то есть

$$
W_{0}>\max \left\{\operatorname{LM}\left(a_{i}^{\prime} u_{i}^{\prime} G_{i}\right) \mid i \in\{1, \ldots, m\} .\right.
$$

Существует элемент $r_{1} \in R^{*}$ такой, что $\operatorname{Lt}\left(a_{1} u_{1} G_{1}\right)=r_{1} W$. Пусть также $r_{2} \in R^{*}-$ такой элемент, что $\dot{G}_{2}=r_{2} G_{2}$. Домножив левую и правую части равенства (35) на $r_{1} U$, получаем, что

$$
a_{1} u_{1} G_{1}-r_{1} r_{2} U V_{2} G_{2}=\sum_{i=1}^{m} r_{1} a_{i}^{\prime} U u_{i}^{\prime} G_{i}
$$

Из формул (26) и (37) следует, что

$$
F=r_{1} a_{1}^{\prime} U u_{1}^{\prime} G_{1}+\left(a_{2} u_{2}+r_{1} r_{2} U V_{2}+r_{1} a_{2}^{\prime} U u_{2}^{\prime}\right) G_{2}+\sum_{i=3}^{m}\left(a_{i} u_{i}+r_{1} a_{i}^{\prime} U u_{i}^{\prime}\right) G_{i}
$$

Эта формула дает представление полинома $F$, причем, как следует из соотношения (36), или параметр этого представления меньше $W$, или мощность множества $T^{\prime}$, определяемого аналогично множеству $T$, меньше мощности $T$. В любом случае приходим к противоречию с минимальностью величин $W$ и $|T|$.

Докажем, что (b), (е) $\Rightarrow$ (i). Пусть $\|F\| \geqslant \alpha_{j}$ и $N_{1}, N_{2} \in \operatorname{Nor}_{\mathscr{S}_{\chi_{0}} \cup \ldots \cup x_{j}}(F)$. Положим $H=N_{1}-N_{2} \in I$. В силу соотношения (7)

$$
\left\|N_{1}\right\|,\left\|N_{2}\right\| \geqslant \alpha_{j}
$$

а значит, $\|H\| \geqslant \alpha_{j}$. Покажем, что $\|H\| \geqslant \alpha_{j+1}$. Допустим противное, то есть пусть

$$
\|H\|<\alpha_{j+1} .
$$

Согласно (b), существует полином $G \in \chi$ такой, что $\operatorname{LM}(G) \mid \operatorname{LM}(H)$. Так как

$$
\|\mathrm{LM}(H)\|=\|H\|<\alpha_{j+1},
$$

то $G \in \chi_{0} \cup \cdots \cup \chi_{j}$. По крайней мере один из коэффициентов $\operatorname{Cf}\left(N_{1}, \operatorname{Lm}(H)\right)$, $\mathrm{Cf}\left(N_{1}, \operatorname{Lm}(H)\right)$ не равен нулю. Без ограничения общности считаем, что $\mathrm{Cf}\left(N_{1}, \operatorname{Lm}(H)\right) \neq 0$. Так как $\left\|\mathrm{Cf}\left(N_{1}, \operatorname{Lm}(H)\right)\right\| \geqslant \alpha_{j}$, полином $N_{1}$ редуцируем с помощью полинома $G$, что противоречит его нормальности. Итак, $\|H\| \geqslant \alpha_{j+1}$. Покажем, что

$$
H \in\left(\pi^{\alpha_{j+1}} \psi_{0} \cup \ldots \cup \pi^{\alpha_{j+1}} \psi_{j} \cup \chi_{j+1} \cup \ldots \cup \chi_{t}\right)
$$

Если $H=0$, то это очевидно. Пусть $H \neq 0$, тогда, согласно (е), полином $H$ обладает $H$-представлением

$$
H=\sum_{i=1}^{m} a_{i} u_{i} G_{i}
$$

Так как

$$
\mathrm{LM}(H)=\max \left\{\operatorname{LM}\left(a_{i} u_{i} G_{i}\right) \mid i \in\{1, \ldots, m\}\right.
$$


то для всех $i \in\{1, \ldots, m\}$

$$
\left\|\mathrm{LM}\left(a_{i} u_{i} G_{i}\right)\right\| \geqslant\|\mathrm{LM}(H)\| \geqslant \alpha_{j+1} .
$$

Значит, $\left\|a_{i}\right\| \geqslant \alpha_{j+1}-\left\|G_{i}\right\|$ для всех $i \in\{1, \ldots, m\}$, что доказывает включение (39).

Докажем, что (i) $\Rightarrow$ (d). Пусть $F \in I$, тогда, так как $I=(\chi)$, справедливо представление

$$
F=\sum_{j=0}^{t} \sum_{s=1}^{m_{j}} a_{s}^{j} u_{s}^{j} G_{s}^{j}
$$

где $G_{s}^{j} \in \chi_{j}$ для всех $s \in\left\{1, \ldots, m_{j}\right\}$. Согласно предложению 1 , существует композиция одношаговых редукщий $\rho_{0} \in \hat{S}_{\chi_{0}}$ такая, что для всех $s \in\left\{1, \ldots, m_{0}\right\}$

$$
\rho_{0}\left(a_{s}^{0} u_{s}^{0} G_{s}^{0}\right) \in \operatorname{Nor}_{\mathscr{C}_{0}}\left(a_{s}^{0} u_{s}^{0} G_{s}^{0}\right)
$$

Так как $0 \in \operatorname{Nor}_{\mathscr{S}_{\chi_{0}}}\left(a_{s}^{0} u_{s}^{0} G_{s}^{0}\right)$ для всех $s \in\left\{1, \ldots, m_{0}\right\}$, согласно (i) для всех $s \in\left\{1, \ldots, m_{0}\right\}$

$$
\rho_{0}\left(a_{s}^{0} u_{s}^{0} G_{s}^{0}\right) \in\left(\pi^{\alpha_{1}} \psi_{0} \cup \chi_{1} \cup \ldots \cup \chi_{t}\right)
$$

С другой стороны, для всех $j \in\{1, \ldots ; t\}$ и $s \in\left\{1, \ldots, m_{j}\right\}$

$$
\rho_{0}\left(a_{s}^{j} u_{s}^{j} G_{s}^{j}\right) \equiv a_{s}^{j} u_{s}^{j} G_{s}^{j} \bmod \left(\pi^{\alpha_{1}} \psi_{0}\right)
$$

Учитывая предложение 8 , из соотношений (42), (44) и (45) получаем, что

$$
\rho_{0}(F)=\sum_{j=0}^{t} \sum_{s=1}^{m_{j}} \rho_{0}\left(a_{s}^{j} u_{s}^{j} G_{s}^{j}\right) \in\left(\pi^{\alpha_{1}} \psi_{0} \cup \chi_{1} \cup \ldots \cup \chi_{t}\right) .
$$

Рассуждая аналогично, находим такую редукцию $\rho_{1} \in \hat{S}_{\chi_{0} \cup \chi_{1}}$, что

$$
\rho_{1} \rho_{0}(F) \in\left(\pi^{\alpha_{2}} \psi_{0} \cup \pi^{\alpha_{2}} \psi_{1} \cup \chi_{2} \cup \ldots \cup \chi_{t}\right)
$$

В итоге мы получим серию редукций $\rho_{0}, \rho_{1}, \ldots, \rho_{t} \in \hat{S}_{\chi}$ такую, что $\rho_{t} \ldots \rho_{1} \rho_{0}(F)=0$. Значит, условие из (d) выполнено.

Доказательство теоремы завершено.

Определение 12. В условиях теоремы 1 систему полиномов $\chi$, удовлетворяющую эквивалентным условиям (a)-(i), назовем стандартным базисом идеала $I$ (согласованным с нормой (6) кольца $R$ ).

Будем говорить, что система полиномов $\chi$ является стандартным базисом, если она является стандартным базисом идеала $\chi R[X]$.

Замечание 5. Ни в теореме 1 , ни в определении 12 не предполагается конечность множества $\chi$. Например, любой идеал $I$ является тривиальным примером собственного стандартного базиса.

В условиях теоремы 1 идеал $I$ может быть нулевым, причем стандартным базисом в этом случае будет система $\{0\}$.

Стандартный базис $\chi$ ненулевого идеала $I$ может содержать 0 . Тем не менее, система $\chi \backslash 0$ также является стандартным базисом $I$. 
Условие (g) теоремы 1 позволяет построить эффективную процедуру, определяющую, явяется ли некоторое конечное множество полиномов $\chi \subset R[X]$ стандартным базисом идеала $(\chi)$. Более того, можно указать алгоритм, находящий стандартный базис идеала, заданного конечной системой порождающих, формально повторяющий известный алгоритм для полей.

Алгоритм 1 (вычисление стандартного базиса).

INPUT: $\varphi=\left\{F_{1}, \ldots, F_{s}\right\} \subset R[X]$

OUTPUT: $\chi=\left\{G_{1}, \ldots, G_{t}\right\}$ - стандартный базис идеала $(\varphi)$

INITIAIIZATION: $\chi:=\psi, \mathscr{G}=\left\{\left(F_{i}, F_{j}\right) \mid 1 \leqslant i<j \leqslant s\right\}$

WHILE $\varphi \neq \varnothing$ DO

Выбираем произвольно $(F, G) \in \mathscr{G}$

$\mathscr{G}:=\mathscr{G} \backslash\{(F, G)\}$

Вычисляем любой элемент $H \in \operatorname{Nor}_{\mathscr{S}_{x}}(S(F, G))$

IF $H \neq 0$ THEN

$\mathscr{G}:=\mathscr{G} \cup\{(U, H) \mid U \in \chi\}$

$\chi:=\chi \cup\{H\}$

Для того, чтобы доказать сходимость представленного алгоритма, нам потребуются дополнительные рассуждения.

Определение 13. Говорят, что упорядоченное множество $(M, \leqslant)$ удовлетворяет условию Хигмана-Коэна, если в любом его бесконечном подмножестве существует бесконечная цепь, то есть бесконечное линейно упорядоченное подмножество.

Несложно доказать следующее утверждение.

Предложение 10. Если на множестве $M$ задан порядок $\leqslant$ удовлетворяющий условию Хигмана-Коэна, то порядок $\leqslant$ на множестве $M^{k}$, задаваемый формулой

$$
\left(a_{1}, \ldots, a_{k}\right) \leqslant\left(b_{1}, \ldots, b_{k}\right) \Longleftrightarrow a_{1} \leqslant b_{1}, \ldots, a_{k} \leqslant b_{k},
$$

также будет удовлетворять условию Хигмана-Коэна.

Очевидно, что естественный порядок $\leqslant$ на $\mathbf{N}$ удовлетворяет условию Хигмана-Коэна. Значит, согласно предложению 10 , порядок $\leqslant$, вводимый на $\mathrm{N}^{k}$ формулой (48), тоже будет удовлетворять условию Хигмана-Коэна.

Как уже отмечалось, упорядоченное множество $\left(\mathbf{N}^{k}, \leqslant\right)$ изоморфно упорядоченному множеству $([X], \mid)$. Значит, отношение делимости | на $[X]$ удовлетворяет условию Хигмана-Коэна.

Из вышесказанного вытекает, что и упорядоченное множество $([\pi, X], \mid)$ удовлетворяет условию Хигмана-Коэна.

Определение 14. Упорядоченное множество $(M, \leqslant)$ называется конечно свободным, если любая его антищепь (то есть подмножество, состоящее из попарно несравнимых элементов) конечна.

Предложение 11. Если упорядоченное множество $(M, \leqslant)$ удовлетворяет условию Хигмана-Коэна, то оно конечно свободно.

Доказательство. Пусть в $M$ существует бесконечная антищепь, согласно условию Хигмана-Коэна в ней можно выделить бесконечную цепь. Получаем противоречие. 
Предложение 12. Упорядоченное множество ([ $\pi, X], \mid)$ конечно свободно. Полугруппа $[\pi, X]$ нетерова (то есть любой ее полугрупповой идеал конечно порожден).

Доказательство. Первое утверждение является непосредственным следствием предложения 11.

Докажем второе утверждение. Пусть $A \triangleleft[\pi, X]$. Рассмотрим множество $Y$ всех минимальных элементов $A$ относительно порядка делимости. Множество $Y$ образует антицепь в $([\pi, X], \mid)$, и следовательно, в силу первого утверждения, оно конечно. Ввиду того, что отношение делимости на $[\pi, X]$ удовлетворяет условию минимальности, выполняется соотношение $A=Y[\pi, X]$.

Теорема 2. Для произвольной непустой системы полиномов

$$
\psi=\left\{F_{1}, \ldots, F_{s}\right\} \subset R[X]
$$

приведенный выше алгоритм 1 за конечное число шагов вычисляет стандартный базис идеала $I=(\psi)$.

Доказательство. Условие (g) теоремы 1 показывает, что наш алгоритм построит стандартный базис. Таким образом, остается проверить, что этот алгоритм завершится за конечное время. В противном случае мы получим бесконечную возрастающую цепь $\operatorname{LM}\left(\chi_{1}\right)[\pi, X] \supset \operatorname{LM}\left(\chi_{2}\right)[\pi, X] \supset \ldots$ идеалов из $[\pi, X]$, что невозможно ввиду второго утверждения предложения 12. (Здесь $\chi_{i+1}$ получается из $\chi_{i}$ путем добавления полинома $H \neq 0$, редуцированного относительно $\left.\chi_{i}\right)$.

Предложение 13. Любой идеал кольча $R[X]$ обладает конечным стандартным базисом.

Доказательство. Так как кольцо $R[X]$ нетерово, любой его идеал имеет конечную систему образующих. Применяя к этой системе образующих алгоритм 1 , получаем конечный стандартный базис исходного идеала. базиса.

В заключение этого пункта исследуем вопрос о возможной избыточности стандартного

Определение 15. Полином $G \in R[X]$ назовем саморедуцированным или самонормальным, если для всех $u \in[X] \backslash\{1\}$

$$
r_{G, u}(G)=G
$$

В противном случае будем говорить, что $G$ является саморедущируемым. Нулевой полином считаем саморедуцированным.

Пример 1. В кольце $\mathrm{Z}_{4}\left[x_{1}, x_{2}\right]$ полином $G=x_{1} x_{2}+1+2 x_{1} x_{2}^{2}$ является саморедуцируемым, так как

$$
r_{G, x_{2}}(G)=G-2 x_{2} G=x_{1} x_{2}+1+2 x_{2} \neq G .
$$

Полученный полином $r_{G, x_{2}}(G)=x_{1} x_{2}+1+2 x_{2}$ самонормален.

Предложение 14. Для любого полинома $G \in R[X]$ существуют самонормальный полином $F \in R[X]$ и обратимый полином $H \in(R[X])^{*}$ такие, что $G H=F$. 
Доказательство. Пусть полином $G \in R[X]$ саморедуцируем. Ясно, что

$$
r_{G, u}(G)=G-b u G=(1-b u) G
$$

для соответствующих $b \in R$ и $u \in[X]$. Так как $u \neq 1$, справедливо неравенство $\|b\| \geqslant 1$, и следовательно, $r_{G, u}(G)=H G$, где $H \in(R[X])^{*}$. Будем производить эти редукщии до получения самонормального полинома. Данный процесс завершится за конечное число шагов, поскольку порядок $\preccurlyeq$ из схемы симплификации $\mathbb{S}_{R[X]}$ (см. раздел 3) удовлетворяет условию обрыва убывающих цепочек, а редукции приводят к уменьшению полинома относительно этого порядка.

В частном случае, когда $G$ - полином от одной переменной нормы 0 , последнее предложение совпадает с известной теоремой Крулля (см. [13] или [1]).

Определение 16. Система полиномов $\chi \subseteq R[X]$ называется редуцированной, если для любого полинома $G \in \chi$

(1) $G$ самонормален,

(2) $G$ нормален относительно схемы симплификации $\mathbb{S}_{\chi} \backslash\{G\}$.

В противном случае будем говорить, что система $\chi$ редуцируема.

Замечание 6. Пусть конечная система $\psi \subset R[X]$ редуцируема, тогда существует полином $G \in \psi$, который или саморедуцируем, или редуцируем относительно системы $\chi \backslash\{G\}$. Заменив полином $G$ в системе $\psi$ на результат его редуцирования, получим новую систему $\psi^{\prime}$. Предложение 14 показывает, что $\psi^{\prime} R[X]=\psi R[X]$. Применив некоторое число раз подобную процедуру, получим редуцированную систему $\chi$ такую, что $\chi R[X]=\psi R[X]$. Этот процесс завершится за конечное число шагов, поскольку порядок $\preccurlyeq$ из схемы симплификации $\mathscr{S}_{R[X]}$ (см. раздел 3) удовлетворяет условию обрыва убывающих цепочек, а редукции приводят к уменьшению полинома относительно этого порядка.

Оформим сказанное выше в виде следующего алгоритма.

Алгоритм 2 (редуцирование систем полиномов).

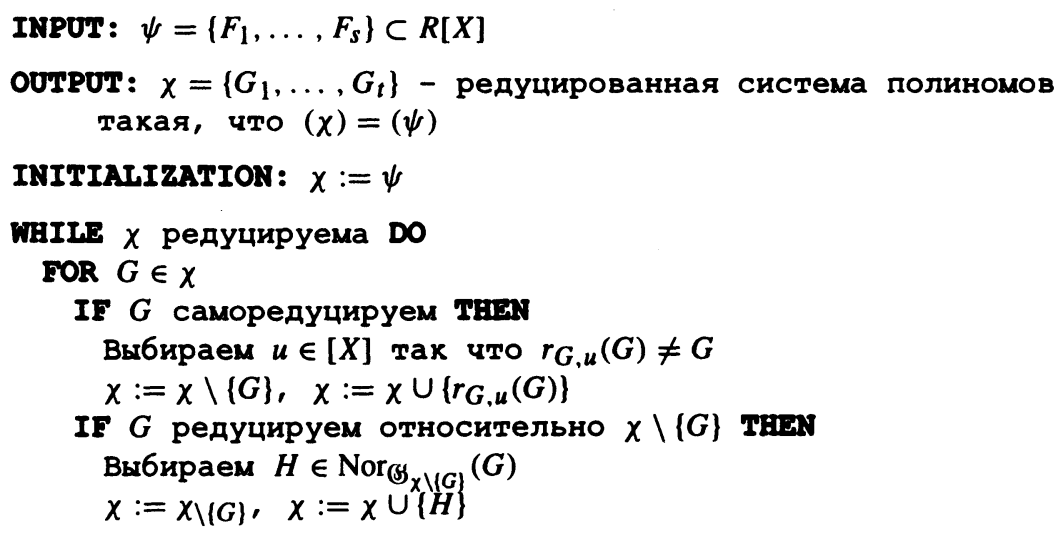

В дальнейшем нам потребуется следующее утверждение. 
Предложение 15. Пусть конечная система полиномов $\psi \subset R[X]$ такова, что множество $\mathrm{LM}(\psi)$ является антицепью относительно порядка делимости, и пусть $\chi$ - результат приведения $\psi к$ редучированному виду. Тогда $\operatorname{LM}(\psi)=\operatorname{LM}(\chi)$.

Доказательство. Так как $\mathrm{LM}(\psi)$ является антицепью, любая редукция не затрагивает ведущих $\pi$-мономов.

Определение 17. Стандартный базис $\chi$ идеала $I$ назовем минимальным, если никакое собственное подмножество $\chi$ не является стандартным базисом идеала $I$.

Определение 18. Стандартный базис $\chi$ идеала $I$ назовем редуцированным, если множество $\chi$ является редуцированной (см. определение 16) системой $\pi$-унитарных полиномов и $0 \notin \chi$, когда $I \neq 0$.

Пусть $(M, \preccurlyeq)$ - упорядоченное множество. Совокупность минимальных элементов $M$ относительно $\preccurlyeq$ будем обозначать $\min (M, \preccurlyeq)$. Ясно, что для любого упорядоченного множества $(M, \preccurlyeq)$ совокупность $\min (M, \preccurlyeq)$ будет антицепью.

Определение 19. Множеством обструкщий идеала $I \triangleleft R[X]$ называется совокупность $\pi$-мономов

$$
O(I)=\min (\operatorname{LM}(I), \mid) .
$$

Отметим, что множество обструкщий неявно зависит от выбора допустимого порядка на $[X]$, так как от этого выбора зависит $\mathrm{LM}(I)$.

Так как отношение делимости удовлетворяет условию минимальности, $O(I)$ всегда непусто. Более того, согласно предложению $12, O(I)$ всегда конечно.

Tеорема 3. Пусть I идеал в $R[X]$, тогда справедливы следующие утверждения.

(a) Система $\chi \subseteq I$ является стандартным базисом $I$, если и только если $O(I) \subseteq \mathrm{LM}(\chi)$.

(b) Стандартный базис х идеала I минимален в том и только том случае, когда $|\chi|=|O(I)|$.

(с) Любой редучированный стандартный базис является минимальным.

(d) Идеал I обладает редучированным стандартным базисом.

Доказательство. Докажем утверждение (а). Пусть $\chi$ - стандартный базис идеала $I$. Если $U \in O(I)$, то $U \in \operatorname{LM}(I)$, и значит, сушествует полином $G \in \chi$ такой, что $\operatorname{LM}(G) \mid U$. Но $U$ - обструкщия, и значит, $\operatorname{LM}(G)=U$. Итак, $U \in \operatorname{LM}(\chi)$.

Обратное включение следует из утверждения (а) теоремы 1 и того факта, что $\operatorname{LM}(I)=O(I)[\pi, X]$.

Утверждение (b) - следствие утверждения (a).

Докажем утверждение (c). Пусть $\chi$ - редуцированный стандартный базис. Ясно, что $\mathrm{LM}(\chi)=O(I)$, иначе система $\chi$ содержала бы 0 при $I \neq 0$ или была бы редуцируемой. Более того, ввиду редуцированности система $\chi$ для каждого $\pi$-монома $U \in O(I)$ содержит ровно один полином $G$ такой, что $\operatorname{LM}(G)=U$.

Докажем утверждение (d). Из произвольного стандартного базиса $\chi$ идеала $I$ (например, можно положить $\chi=I$ ), получим минимальный стандартный базис, выбрав из $\chi$ для каждого $U \in O(I)$ ровно по одному полиному с ведущим $\pi$-мономом, равным $U$ (см. (a) и (b)). Любой минимальный стандартный базис путем последовательных редукций может быть приведен к редуцированному стандартному базису (см. замечание 6). Таким образом, идеал $I \triangleleft R[X]$ обладает редуцированным стандартным базисом. 
Следующее предложение показывает, насколько однозначно определены параметры минимальных и редуцированных стандартных базисов. Отметим, что аналогичные утверждения были доказаны ранее для канонической системы образующих (см. [1-3]).

Предложение 16. Пусть $\chi=\chi_{0} \cup \ldots \cup \chi_{t} u \varphi=\varphi_{0} \cup \ldots \cup \varphi_{s}-$ минимальные стандартные базисы идеала I, представленные в виде (30) со свойством (31). Тогда $t=s,\left|\chi_{i}\right|=\left|\varphi_{i}\right|$ $u\left\|\chi_{i}\right\|=\left\|\varphi_{i}\right\|=\alpha_{i}, i \in\{0,1, \ldots, t\}$.

Если, кроме того, стандартные базисы $\chi и \varphi$ являются редучированными, то $\chi_{i} \equiv \varphi_{i}\left(I \cap \pi^{\alpha_{i+1}} R[X]\right), i \in\{0,1, \ldots, t\}$.

Доказательство. Первая часть данного предложения является следствием теоремы 3 , согласно которой $\mathrm{LM}(\chi)=\operatorname{LM}(\varphi)=O(I)$ и $|\chi|=|\varphi|=|O(I)|$.

Пусть теперь $\chi$ и $\varphi$ - редуцированные стандартные базисы и полиномы $F \in \chi_{i}$ и $G \in \varphi_{i}$ таковы, что $\operatorname{LM}(F)=\operatorname{LM}(G)=U$. Их можно представить в виде $F=U+F_{0}$ и $G=U+G_{0}$. Разность $H=F-G=F_{0}-G_{0}$ принадлежит идеалу $I \cap \pi^{\alpha_{i}} R[X]$. Покажем, что $H \in \pi^{\alpha_{i+1}} R[X]$. Допустим противное, то есть пусть $\|H\|<\alpha_{i+1}$. Существует обструкция $V \in O(I)$ такая, что $V \mid \operatorname{LM}(H)$. Справедливо неравенство $\|\mathrm{LM}(H)\|=\|H\|<\alpha_{i+1}$, поэтому $\|V\| \leqslant \alpha_{i}$. По крайней мере один из многочленов $F_{0}$ или $G_{0}$ содержит ненулевой член, делящийся на $V$, что противоречит редуцированности базисов $\chi$ и $\varphi$. Значит, $\|H\| \geqslant \alpha_{i+1}$, и следовательно, $F-G=H \in I \cap \pi^{\alpha_{i+1}} R[X]$.

Пример 2. Отметим, что редуцированный стандартный базис определяется, вообще говоря, неоднозначно. Например, для $R[X]=\mathbf{Z}_{4}\left[x_{1}\right]$ системы полиномов $\left\{x_{1}^{2}+x_{1}, 2 x_{1}\right\}$ и $\left\{x_{1}^{2}+3 x_{1}, 2 x_{1}\right\}$ являются редуцированными стандартными базисами одного и того же идеала.

\section{5. Свойства стандартных базисов. Связь с канонической системой образующих}

В следующем предложении устанавливаются свойства стандартных базисов, аналогичные свойствам канонической системы образующих из [3] (см. теорему 4.11 в [3]).

Предложение 17. Пусть $\chi=\chi_{0} \cup \ldots \cup \chi_{t}-$ стандартный базис идеала I, представленный в виде (30) со свойством (31). Выберем системы полиномов $\psi_{j}, j \in\{0,1, \ldots, t+1\}$ так, чтобы выполнялось условие (32). Пусть также дано число $a \in\{0,1, \ldots, n\}$ u $j_{0}=\min \left\{j \in\{0,1, \ldots, t+1\} \mid a \leqslant \alpha_{j}\right\}$. Тогдa

(a) $\chi_{0} \cup \ldots \cup \chi_{j_{0}-1} \cup\left\{\pi^{a}\right\}-$ стандартный базис идеала $I+\pi^{a} R[X]$,

(b) $\psi_{0} \cup \ldots \cup \psi_{j_{0}-1} \cup \pi^{\alpha_{j_{0}}-a} \psi_{j_{0}} \cup \ldots \cup \pi^{\alpha_{t}-a} \psi_{t} \cup\left\{\pi^{n-a}\right\}-$ стандартный базис идеала $\left(I: \pi^{a}\right)$,

(c) $\pi^{a} \psi_{0} \cup \ldots \cup \pi^{a} \psi_{j_{0}-1} \cup \chi_{j_{0}} \cup \ldots \cup \chi_{t}-$ стандартный базис идеала $I \cap \pi^{a} R[X]$.

Доказательство. Очевидно, что соответствующие системы полиномов лежат в соответствующих идеалах. Поэтому достаточно проверить, что в каждом рассматриваемом случае выполняется условие (b) теоремы 1.

Докажем утверждение (а). Пусть $F \in I+\pi^{a} R[X]$. Тогда $F=H+\pi^{a} L$, где $H \in I$ и $L \in R[X]$. Если $\|H\| \geqslant a$, то $\pi^{a} \mid \operatorname{LM}(F)$. Если $\|H\|<a$, то существует полином $G \in \chi_{0} \cup \ldots \cup \chi_{j_{0}-1}$ такой, что $\operatorname{LM}(G) \mid \operatorname{LM}(H)$. Но $\operatorname{LM}(F)=\operatorname{LM}(H)$, и значит, $\operatorname{LM}(G) \mid \operatorname{LM}(F)$. 
Докажем утверждение (b). Пусть $F \in\left(I: \pi^{a}\right)$. Тогда $H=\pi^{a} F \in I$. Если $\|F\| \geqslant n-a$, то $\pi^{n-a} \mid \mathrm{LM}(F)$. Пусть $\|F\|<n-a$. Найдется полином $G \in \chi_{j}$ такой, что $\operatorname{LM}(G) \mid \mathrm{LM}(H)$. Его можно представить в виде $G=\pi^{\alpha_{j}} G_{0}$, где $G_{0} \in \psi_{j}$. Ясно, что $\pi^{\alpha_{j}} \operatorname{LM}\left(G_{0}\right) \mid \pi^{a} \operatorname{LM}(F)$. Так как $\pi^{a} \operatorname{LM}(F) \neq 0$, то $\operatorname{LM}\left(G_{0}\right) \mid \operatorname{LM}(F)$ при $j<j_{0}$ и $\pi^{\alpha_{j}-a} \operatorname{LM}\left(G_{0}\right) \mid \mathrm{LM}(F)$ при $j \geqslant j_{0}$.

Докажем утверждение (c). Пусть $F \in I \cap \pi^{a} R[X]$. Существует полином $G \in \chi_{j}$ такой, что $\operatorname{LM}(G) \mid \operatorname{LM}(F)$. Если $j \geqslant j 0$, то требуемое доказано. Пусть $j<j 0$, представим полином $G$ в виде $G=\pi^{\alpha_{j}} G_{0}$, где $G_{0} \in \psi_{j}$, тогда $\pi^{a} \operatorname{LM}\left(G_{0}\right) \mid \operatorname{LM}(F)$, но $\pi^{a} \operatorname{LM}\left(G_{0}\right)=\operatorname{LM}\left(\pi^{a} G_{0}\right)$.

В [3] была введена каноническая система образующих (КСО) идеала $I \triangleleft R[X]$. Установим связь между КСО и введенным в разделе 4 понятием стандартного базиса.

Нам будет удобно дать определение КСО, используя обозначения и терминологию данной статьи.

Определение 20 ([3]). Система $\varphi=\varphi_{0} \cup \ldots \cup \varphi_{t}$ полиномов из идеала $I \neq 0$ называется канонической системой образующих (КСО) $I$, если она непуста, не содержит 0 и выполнены следующие условия:

- для любого $j \in\{0,1, \ldots, t\}$ система $\varphi_{j}$ является редуцированной,

$-\operatorname{Lm}\left(\varphi_{0}\right)[X] \subsetneq \ldots \subsetneq \operatorname{Lm}\left(\varphi_{t}\right)[X]$

- для любых $F \in I$ и $j \in\{0,1, \ldots, t\}$ имеет место импликация

$$
\left(\operatorname{supp}(F) \subseteq[X] \backslash \operatorname{Lm}\left(\varphi_{j}\right)[X]\right) \Longrightarrow\left(\|F\| \geqslant\left\|\varphi_{j+1}\right\|\right) .
$$

Предложение 18. Любая КСО ненулевого идеала $I \triangleleft R[X]$ является его стандартным базисом.

Доказательство. Пусть $\varphi=\varphi_{0} \cup \ldots \cup \varphi_{t}-\mathrm{KCO} \mathrm{I,} \mathrm{и} \mathrm{пусть} F \in I$. Известно ([3], теорема 4.10), что $I=\varphi R[X]$, и стало быть $\|F\| \geqslant\left\|\varphi_{0}\right\|=\|I\|$. Пусть $j_{0}$ - наибольшее из чисел $j \in\{0,1, \ldots, t\}$ таких, что $\|F\| \geqslant\left\|\varphi_{j}\right\|$.

Если $j_{0}=t$, то $F=0$ и $\operatorname{LM}(F)=0 \in \operatorname{LM}(\varphi)[\pi, X]$.

Пусть $j_{0}<t$. Если $\operatorname{Lm}(F) \in \operatorname{Lm}\left(\varphi_{j_{0}}\right)[X]$, то существует полином $G \in \varphi_{j_{0}}$ такой, что $\operatorname{Lm}(G) \mid \operatorname{Lm}(F)$, и так как $\|F\| \geqslant\left\|\varphi_{j_{0}}\right\|=\|G\|$, справедливо соотношение $\operatorname{LM}(G) \mid \operatorname{LM}(F)$.

Остается показать, что случай $\operatorname{Lm}(F) \notin \operatorname{Lm}\left(\varphi_{j_{0}}\right)[X]$ невозможен. Допустим противное, тогда $\operatorname{Lm}(F) \in[X] \backslash \operatorname{Lm}\left(\varphi_{j_{0}}\right)[X]$, и значит, $\operatorname{Lt}(F)$ нормален относительно схемы симплификации $\mathscr{S}_{\varphi_{j_{0}}}$. Запишем $F$ в виде

$$
F=\operatorname{Lt}(F)+H
$$

Ясно, что $\operatorname{Lt}(F) \succ H$. Пусть $\rho \in S_{\Theta_{\varphi_{j_{0}}}}$ - такая редукщия, что полином $\rho(H)$ нормален относительно $\mathbb{S}_{\varphi_{j_{0}}}$. В силу предложения 8

$$
\rho(F)=\rho(\operatorname{Lt}(F))+\rho(H)=\operatorname{Lt}(F)+\rho(H) .
$$

Из соотношения $\operatorname{Lt}(F) \succ H \succcurlyeq \rho(H)$ вытекает равенство $\operatorname{LM}(F)=\operatorname{LM}(\rho(F))$, и стало быть $\|\rho(F)\|=\|F\|$. С другой стороны, $\operatorname{supp}(\rho(F)) \subseteq[X] \backslash \operatorname{Lm}\left(\varphi_{j_{0}}\right)[X]$, и значит, согласно определению $20,\|\rho(F)\| \geqslant\left\|\varphi_{j_{0}+1}\right\|>\|F\|$. Полученное противоречие завершает доказательство. 
Пусть $\chi=\chi_{0} \cup \ldots \cup \chi_{t}-$ минимальный стандартный базис идеала $I \neq 0$, представленный в виде (30) со свойством (31). Мы покажем, как, исходя из $\chi$, можно построить КСО идеала $I$.

Построим по индукщии семейства полиномов $\varphi_{0}, \ldots, \varphi_{t}$. Пусть $\varphi_{0}-$ результат редуцирования (см. замечание 6) системы $\chi_{0}$. Предположим, что семейство $\varphi_{j}, j \in\{0, \ldots, t-1\}$ уже задано, определим $\varphi_{j+1}$. Пусть

$$
\varphi_{j+1}^{\prime}=\left\{G \in \pi^{\alpha_{j+1}-\alpha_{j}} \varphi_{j} \mid \operatorname{LM}(G) \notin \operatorname{LM}\left(\chi_{j+1}\right)[\pi, X]\right\}
$$

Систему $\varphi_{j+1}$ определим, как результат приведения $\chi_{j+1} \cup \varphi_{j+1}^{\prime}$ к редуцированному виду. Лемма 2. Для любого $j \in\{0,1, \ldots, t\}$ верно равенство

$$
\operatorname{LM}\left(\varphi_{j}\right)=\min \left(\operatorname{LM}\left(\pi^{\alpha_{j}-\alpha_{0}} \chi_{0} \cup \pi^{\alpha_{j}-\alpha_{1}} \chi_{1} \cup \ldots \cup \chi_{j}\right), \mid\right)
$$

Доказательство. Применим индукцию по $j \in\{0,1, \ldots, t\}$. При $j=0$ в силу предложения 15 и минимальности базиса $\chi$

$$
\operatorname{LM}\left(\varphi_{0}\right)=\operatorname{LM}\left(\chi_{0}\right)=\min \left(\operatorname{LM}\left(\chi_{0}\right), \mid\right)
$$

Пусть утверждение верно при $j \in\{0,1, \ldots, t-1\}$, покажем, что оно справедливо и для $j+1$. Из формулы (50) следует, что

$$
\operatorname{LM}\left(\varphi_{j+1}^{\prime}\right)=\operatorname{LM}\left(\pi^{\alpha_{j+1}-\alpha_{j}} \varphi_{j}\right) \backslash \operatorname{LM}\left(\chi_{j+1}\right)[\pi, X],
$$

и значит, $\operatorname{LM}\left(\varphi_{j+1}^{\prime}\right)$ совпадает с $\min \left(\operatorname{LM}\left(\pi^{\alpha_{j+1}-\alpha_{0}} \chi_{0} \cup \ldots \cup \pi^{\alpha_{j+1}-\alpha_{j}} \chi_{j}, \mid\right) \backslash \operatorname{LM}\left(\chi_{j+1}\right)[\pi, X]\right.$. Учитывая минимальность базиса $\chi$, получаем, что

$$
\operatorname{LM}\left(\varphi_{j+1}^{\prime} \cup \chi_{j+1}\right)=\min \left(\operatorname{LM}\left(\pi^{\alpha_{j+1}-\alpha_{0}} \chi_{0} \cup \ldots \cup \pi^{\alpha_{j+1}-\alpha_{j}} \chi_{j} \cup \chi_{j+1}\right), \mid\right) .
$$

Последнее равенство вместе с предложением 15 завершает шаг индукщии.

Предложение 19. В введенных выше обозначениях система полиномов

$$
\varphi=\varphi_{0} \cup \ldots \cup \varphi_{t}
$$

есть КСО идеала I.

Доказательство. Лемма 2 показывает, что для любого $j \in\{0,1, \ldots, t\}$ множество $\varphi_{j}$ является $\pi$-однородной системой нормы $\alpha_{j}$.

Проверим условия из определения 20.

Первое условие верно по построению системы $\varphi$.

Докажем справедливость второго условия. Из. леммы 20 вытекает равенство

$$
\operatorname{Lm}\left(\varphi_{j}\right)=\min \left(\operatorname{Lm}\left(\chi_{0} \cup \chi_{1} \cup \ldots \cup \chi_{j}\right), \mid\right),
$$

и стало быть,

$$
\operatorname{Lm}\left(\varphi_{j}\right)[X]=\operatorname{Lm}\left(\chi_{0} \cup \chi_{1} \cup \ldots \cup \chi_{j}\right)[X]
$$

Учитывая минимальность базиса $\chi$, получаем требуемое. 
Проверим справедливость третьего условия. Пусть полином $F \in I$ таков, что $\operatorname{supp}(F) \subseteq[X] \backslash \operatorname{Lm}\left(\varphi_{j}\right)[X]$. Так как $\chi-$ стандартный базис идеала $I$, существует полином $G \in \chi$ такой, что $\operatorname{LM}(G) \mid \mathrm{LM}(F)$. Вместе с тем, как следует из формулы (52), $F$ нормален относительно $\mathscr{S}_{\chi_{0} \cup \ldots \cup \chi_{j}}$. Следовательно, $G \in \chi_{j+1} \cup \ldots \cup \chi_{t}$, и значит,

$$
\|F\|=\|\operatorname{LM}(F)\| \geqslant\|\operatorname{LM}(G)\|=\|G\| \geqslant \alpha_{j+1}=\left\|\psi_{j+1}\right\|,
$$

что и требовалось доказать.

Для подтверждения того, что понятия КСО и минимального (редуцированного) стандартного базиса не совпадают, рассмотрим следующий пример.

Пример 3. Пусть $R[X]=Z_{4}\left[x_{1}, x_{2}\right]$ и в качестве допустимого порядка на $[X]=\left[x_{1}, x_{2}\right]$ используется лексикографический порядок с $x_{1}>x_{2}$. Система полиномов $\varphi=\left\{x_{1}^{2}, x_{2}^{2}+2 x_{2}, 2 x_{1}^{2}, 2 x_{2}\right\}$ есть КСО порождаемого ею идеала, вместе с тем этот идеал имеет минимальный (редуцированный) стандартный базис $\chi=\left\{x_{1}^{2}, x_{2}^{2}, 2 x_{2}\right\}$, состоящий из меньшего, чем $\varphi$, числа полиномов.

Тем не менее, КСО может быть редуцированным стандартным базисом. Например, $\varphi=\left\{x_{1}^{2}, x_{2}^{2}, 2 x_{1}, 2 x_{2}\right\}$ является одновременно КСО и редуцированным стандартным базисом идеала $(\varphi)$.

\section{6. Цилиндрические идеалы и поднятия}

Напомним некоторые определения из теории модулей. Пусть $T$ - произвольное кольцо и $M_{T}$ - правый $T$-модуль. Подмодуль $A \leqslant M_{T}$ называется существенным подмодулем в $M_{T}$, если для любого $B \leqslant M_{T}$ из $A \cap B=0$ следует, что $B=0$. Подмодуль $B \leqslant M_{T}$ называется дополнением по пересечению для подмодуля $A \leqslant M_{T}$, если $A \cap B=0$ и для любого $C \leqslant M_{T}$ из $A \cap C=0$ и $B \leqslant C$ вытекает, что $C=B$. Мономорфизм $\mu: M \rightarrow I(M)$ называется инъективной оболочкой модуля $M$, если $I(M)$ - инъективный модуль и образ $\mu$ существенен в $I(M)$.

Далее мы используем следующий результат (см., например, [14]).

Теорема 4. Пусть $T$ - произвольное кольчо, $M$ - правый $T$-модуль $и \mathrm{I}(M)-$ его инъективная оболочка. Тогда для любого подмодуля $X \leqslant M T$, следующие условия эквивалентны:

(а) $X$ является замкнутым подмодулем в $M$, то есть для всех $U \leqslant M$

$$
(X-\text { существенный подмодуль } U) \Longrightarrow(X=U),
$$

(b) $X$ является дополнением по пересечению некоторого подмодуля в $M_{T}$,

(c) для любого существенного подмодуля $V \leqslant M_{T}$ такого, что $X \leqslant V$, модуль $V / X$ существенен в $M / X$, то есть естественное отображение $\nu: M \rightarrow M / X$ сохраняет суиественные подмодули.

(d) $X$ представим в виде $X=M \cap Q$, где $Q$ - прямое слагаемое в $\mathrm{I}(M)$.

В нашем случае $(T=R)$ теорему 4 можно дополнить еще одним условием.

Предложение 20. Пусть $M-$ правый $R$-модуль. Тогда его подмодуль $X \leqslant M_{R}$ замкнут в том и только том случае, когда $X \pi=X \cap M \pi$. 
Доказательство. Докажем импликацию $\Rightarrow$. Пусть $X$ замкнут в $M_{R}$. Очевидно, $X \pi \leqslant X \cap M \pi$. Докажем, что любой $z=m \pi \in X \cap M \pi$ принадлежит $X \pi$. Если $m \in X$, то это очевидно. Допустим, что $m \in M \backslash X$.

Докажем, что модуль $(m R+X) / X$ прост. Пусть $m r+X$ - его произвольный ненулевой элемент. Тогда $m r \notin X$, и по предположению $r \in R^{*}$. Следовательно, $m+X \in(m r+X) R$ и $(m r+X) R=(m R+X) / X$, что и требуется.

Согласно пункту (b) теоремы 4 , подмодуль $X$ есть дополнение по пересечению некоторого подмодуля $A \leqslant M_{R}$. Значит, фактормодуль $(X+A) / X$ является существенным подмодулем в $M / X$, и следовательно, $(m R+X) / X \leqslant(X+A) / X$. Таким образом, $m \in X \oplus A$, то есть $m=x+a$ для $x \in X$ и $a \in A$. Домножая на $\pi$ и учитывая включение $m \pi \in X$, находим, что $a \pi=0$, и значит, $z=m \pi=x \pi \in X \pi$.

Докажем импликацию $\Leftarrow$. Пусть $X \pi=X \cap M \pi$ и $X$ тем не менее не замкнут. Тогда существует подмодуль $U \leqslant M_{R}$ такой, что $X$ - собственный существенный подмодуль $U$. Сушествует элемент $u \in U \backslash X$ со свойством $u \pi \in X$. По предположению $u \pi=x \pi$ для некоторого $x \in X$. Элемент $a=u-x \in U \backslash X$ таков, что $a \pi=0$. Значит, $X \cap a R=0$, и следовательно, $a=0$, получаем противоречие.

Предложение 21. Пусть $R$ - коммутативное артиново чепное кольчо. Для модуля $M_{R}$ следуюшие условия эквивалентны:

(a) $M_{R}$ свободен,

(b) $M_{R}$ проективен,

(c) $M_{R}$ инъективен,

(d) $M_{R}$ является плоскии,

(e) $\operatorname{Ann}_{M}\left(\pi^{n-1}\right)=M \pi$.

Доказательство. Эквивалентность (a) $\Longleftrightarrow$ (b) следует из локальности кольца $R$.

Эквивалентность (b) $\Longleftrightarrow$ (c) следует из того, что $R$ - квазифробениусово кольцо (см., например, 13.6.1 в [14]).

Докажем, что (b) $\Longleftrightarrow$ (d). Кольцо $R$ артиново и, следовательно, совершенно, а модуль над совершенным кольцом проективен тогда и только тогда, когда он является плоским (см., например, 11.6.3 в [14]).

Эквивалентность (c) мep, 5.7.1 в [14]).

Tеорема 5. Для произвольного идеала $I \triangleleft R[X]$ следующие условия эквивалентны:

(a) $I$ - замкнутый подмодуль $R[X]_{R}$,

(b) I выделяется прямым слагаемым в $R[X]_{R}$,

(c) I является свободным $R$-модулем,

(d) $R[X] / I$ является свободным $R$-модулем,

(e) $\pi I=I \cap \pi R[X]$,

(f) $I=0$ или I обладает $\pi$-однородным стандартным базисом нормы 0. 
Доказательство. Докажем, что (a) $\Longleftrightarrow$ (b). Согласно предложению 21 , модуль $R_{R}$ инъективен. Поскольку кольцо $R$ нетерово, инъективным является и модуль $R[X]_{R} \cong R_{R}^{(\mathrm{N})}$. Таким образом, $\mathrm{I}(R[X])=R[X]$ и требуемое вытекает из теоремы 4.

Эквивалентности (b)

Эквивалентность (a) $\Longleftrightarrow$ (e) есть следствие предложения 20.

Докажем, что (e) $\Rightarrow(\mathrm{f})$. Пусть $I \neq 0$, тогда $\|I\|=0$. Выберем в $\bar{I}$ какой-нибудь стандартный базис $\psi$ и найдем $\pi$-однородную систему $\chi$ нормы 0 такую, что $\bar{\chi}=\psi$. Утверждается, что $\chi$ будет искомым стандартным базисом.

Покажем, что для системы $\chi$ выполняется условие (c) теоремы 1 . Пусть $F \in I$ и $H \in \operatorname{Nor}_{\mathscr{S}_{\chi}}(F)$. Ясно, что $\operatorname{supp}(H) \subseteq \mathscr{F}=[X] \backslash \operatorname{Lm}(\chi)[X]$, и так как $\bar{\chi}-$ базис Гребнера, $\|H\| \geqslant 1$. По предположению $H=\pi F_{1}$, где $F_{1} \in I$. Представим $F_{1}$ в виде $F_{1}=F_{1}^{(0)}+F_{1}^{(1)}$, где $\operatorname{supp}\left(F_{1}^{(0)}\right) \subseteq \mathscr{F}_{\text {и }} \operatorname{supp}\left(F_{1}^{(1)}\right) \cap F_{F}=\varnothing$. Очевидно, что $F_{1}^{(0)}$ нормален относительно $\mathbb{S}_{\chi}$ и $\left\|F_{1}^{(1)}\right\| \geqslant n-1$. Пусть $\rho \in \hat{S}_{\chi}-$ редукщия, приводящая $F_{1}^{(1)}$ к нормальной форме. Справедливы соотношения

$$
\rho\left(F_{1}\right)-F_{1}=\rho\left(F_{1}^{(1)}\right)-F_{1}^{(1)} \in I \cap \pi^{n-1} R[X] .
$$

Так как $\operatorname{supp}\left(\rho\left(F_{1}\right)\right) \subseteq F_{\text {и }} \rho\left(F_{1}\right) \in I$, то $\rho\left(F_{1}\right) \in I \cap \pi R[X]=\pi I$ и стало быть $\rho\left(F_{1}\right)=\pi F_{2}$, где $F_{2} \in I$. Окончательно получаем, что

$$
H=\pi F_{1}=\pi \rho\left(F_{1}\right)=\pi^{2} F_{2}
$$

Рассуждая аналогично, можно показать, что $H=0$.

Импликация (f) $\Rightarrow$ (e) является следствием предложения 17.

Отметим, что в [2] для случая унитарного идеала I было доказано, что КСО I (см. определение 20) является $\pi$-однородной системой нормы 0 в том и только том случае, когда $R[X] / I$ - свободный $R$-модуль (см. 4.15 в [2]), то есть фактически была доказана эквивалентность (d)

Если идеал $I \triangleleft R[X]$ обладает $\pi$-однородным стандартным базисом $\chi$ нормы 0 , то множество нормальных относительно схемы симплификация $\mathbb{S}_{\chi} \pi$-мономов $[\pi, X] \backslash \operatorname{LM}(\chi)[\pi, X]$ совпадает с множеством $([X] \backslash \operatorname{Lm}(\chi)[X])\left\{1, \pi, \ldots ; \pi^{n-1}\right\}$, которое представляется нам цилиндром с основанием $[X] \backslash \operatorname{Lm}(\chi)[X]$, поэтому идеалы, для которых выполняются эквивалентные условия теоремы 5, мы будем называть цилиндрическими.

Пусть $I \triangleleft R[X]-$ унитарный идеал и $\chi \subseteq I$ - редуцированная $\pi$-однородная система полиномов нормы 0 такая, что $\bar{\chi}-$ редуцированный базис Гребнера идеала $\bar{I}$, то есть в определения работы [2] $\chi$ - Круллева система. В [2] было доказано, что из свободы $R$-модуля $R[X] / I$ следует, что $\mathscr{\mho}_{\chi}-$ схема симплификации с канонизацией. Там же был поставлен вопрос об обращении этой импликации. Утверждается, что ответ на этот вопрос положителен. Действительно, $\chi$ - стандартный базис идеала $I$ (теорема 1 ), являющийся $\pi$-однородной системой нормы 0 . Следовательно, по теореме $5 R[X] / I-$ свободный $\boldsymbol{R}$-модуль.

Определение 21. Будем говорить, что базис Гребнера $\psi \subseteq \bar{R}[X]$ поднимается (в $R[X])$, если существует $\pi$-однородная система $\chi \subseteq R[X]$ нормы 0 , являющаяся стандартным базисом, такая, что $\bar{\chi}=\psi$.

При этом систему $\chi$ будем называть поднятием системы $\psi$. 
Теорема 6. Для любого ненулевого идеала $J \triangleleft \bar{R}[X]$ следуюшие условия эквивалентны:

(а) любой базис Гребнера идеала J поднимается,

(b) существует базис Гребнера идеала J, который поднимается,

(c) существует чилиндрический идеал $I \triangleleft R[X]$ такой, что $\bar{I}=J$.

Доказательство. Импликация (a) $\Rightarrow$ (b) очевидна. Импликация (b) $\Rightarrow$ (c) является следствием пункта (g) теоремы 5. Докажем, что (c) $\Rightarrow$ (a). Согласно теореме 5 справедливо равенство $\pi I=I \cap \pi R[X]$. Остается слово в слово повторить доказательство импликации (e) $\Rightarrow$ (f) из теоремы 5 .

Автор глубоко благодарен А. А. Нечаеву и А. В. Михалеву за детальное обсуждение результатов работы и многочисленные важные замечания.

\section{Список литературы}

1. Нечаев А. А., Линейные рекуррентные последовательности над коммутативными кольцами. Дискретная математика (1991) 3, № 4, 105-127.

2. Нечаев А. А., Михайлов Д. А., Каноническая система образующих унитарного полиномиального идеала над коммутативным артиновым цепным кольцом. Дискретная математика (2001) 13, №4, 3-42.

3. Нечаев А. А., Михайлов Д. А., Использование КСО для решения систем уравнений над кольцами. XXII Межсведомств. научно-техн. конф. «Проблемы обеспечения эффективности и устойчивости функчионирования сложных технических систем». СВИ РВ, Серпухов, 2003, т. 2, 4952.

4. Латышев В. Н., Комбинаторная теория колеч, стандартные базисы. Изд-во МГУ, Москва, 1988.

5. Adams W., Loustaunau P., An introduction to Gröbner bases. Amer. Math. Soc., Providence, 1994.

6. Кокс Д., Литтл Дж., О’Ши Д., Идеалы, многообразия и алгоритмы. Мир, Москва, 2000.

7. Norton G. H., Salagean A., Strong Gröbner bases and cyclic codes over a finite-chain ring. Proc. Intern. Workshop on Coding and Cryptography. Paris, 2001, 8-12.

8. Byrne E., Fitzpatrick P., Gröbner bases over Galois rings with an application to decoding alternant codes. J. Symbolic Comput. (2001) 31, 565-584.

9. Robbiano L., On the theory of graded structures. J. Symbolic Comput. (1986) 2, 139-170.

10. Mora T., Seven variations on standard bases. Preprint, Univ. de Genova, Dip. di Math., №45, 1986.

11. Apel J., Computational ideal theory in finitely generated extension rings. J. Theoret. Computer Sci. (2000) 244, 1-33.

12. Kronecker L., Vorlesungen über Zahlentheorie. Bd. 1, Teubner, Leipzig, 1901.

13. Krull W., Algebraische Theorie der Ringe. II. Math. Ann. (1923) 91, 1-46.

14. Каш Ф. Модули и кольча. Мир, Москва, 1981.

Статья поступила 10.11.2003. 